\title{
Axion Searches in the Past, at Present, and in the Near Future
}

\author{
Rémy Battesti ${ }^{1}$, Berta Beltrán ${ }^{2}$, Hooman Davoudiasl ${ }^{3}$, Markus Kuster ${ }^{4,5, *}$, \\ Pierre Pugnat ${ }^{6}$, Raoul Rabadan ${ }^{7}$, Andreas Ringwald ${ }^{8}$, Neil Spooner ${ }^{9}$, and \\ Konstantin Zioutas 6,10 \\ 1 Laboratoire National des Champs Magnétiques Pulsés, CNRS/INSA/UPS, \\ UMR 5147, 31400 Toulouse, France \\ 2 Department of Physics, Queen's University, Kingston, Ontario K7L 3N6, \\ Canada \\ 3 Department of Physics, University of Wisconsin, Madison, WI 53706, USA \\ 4 Technische Universität Darmstadt, IKP, Schlossgartenstrasse 9, D-64289 \\ Darmstadt, Germany \\ 5 Max-Planck-Institut für extraterrestrische Physik, Giessenbachstrasse, D-85748 \\ Garching, Germany \\ ${ }^{6}$ European Organization for Nuclear Research (CERN), CH-1211 Genève 23, \\ Switzerland \\ 7 Institute for Advanced Study, Einstein Drive, Princeton, NJ, 08540, USA \\ 8 Deutsches Elektronen-Synchrotron DESY, Notkestr. 85, D-22607 Hamburg, \\ Germany \\ 9 University of Sheffield, Sheffield S3 7RH, UK \\ 10 University of Patras, Rio, 26500 Patras, Greece \\ *markus.kuster@cern.ch
}

Summary. Theoretical axion models state that axions are very weakly interacting particles. In order to experimentally detect them, the use of colorful and inspired techniques becomes mandatory. There is a wide variety of experimental approaches that were developed during the last 30 years, most of them make use of the Primakoff effect, by which axions convert into photons in the presence of an electromagnetic field. We review the experimental techniques used to search for axions and will give an outlook on experiments planned for the near future.

\section{Searches for Dark Matter Axions}

As described in the review by P. Sikivie on cosmological axions in Chap. ??, axions produced in the early universe with a mass ranging from $\mu \mathrm{eV}$ to meV could account for the cold dark matter component of the universe and therefore an experimental evidence for axions in this mass range would be of great interest nowadays. Microwave resonant cavity experiments are the most 


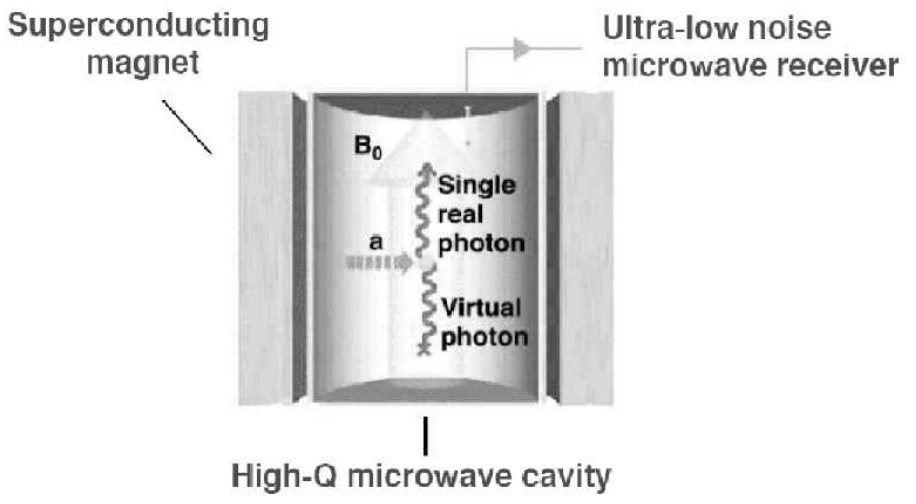

Fig. 1. Schematic principle of the microwave cavity experiment to look for cold dark matter axions. First an axion would be resonantly converted into a quasimonochromatic photon in the magnetic-field permeated microwave cavity. Then an ultra-low-noise receiver is set to detect this photon as an excess on the cavity power output

sensitive detectors for cold-dark matter axions at present. The heart of such a device is a metallic conductor which acts as a cavity for electromagnetic fields such as photons. By tuning the size of the magnetic-field permeated cavity its resonant frequency can be shifted such that it matches the frequency of the axion field (which is related to the axion mass). In this case axions will convert resonantly into quasi-monochromatic photons which could be detected with sensitive microwave receivers as an excess on the cavity power output. In Fig. 1 the schematic working principle of this kind of detectors is shown.

The signal obtained would carry information not only about the axion mass, but also about the axion distribution in the galactic halo, as its width would represent the virial distribution of thermalized axions. Furthermore, the signal may also posses a finer substructure due to axions that have recently fallen into the galaxy and have not yet thermalized, as shown in Fig. 2. Again the reader is refered to Skivie's review for a more detailed description of the axion distribution in the galactic halo.

Due to the very low mass range of the dark matter axion, the expected signal is minuscule and therefore the sensitivity of the experiment crucially depends on the quality of the microwave receivers (see Chap. ?? by Carosi and van Bibber). In the 80s two pioneering experiments were carried out at Rochester-Brookhaven-Fermilab (RBF) (1) and the University of Florida (UF) (2) using small volume cavities $(\sim 11)$ and HFET amplifiers, but their sensitivities were lacking by two or three orders of magnitude the sensitivity necessary to probe the theoretically motivated region in the axion parametric space.

An improvement in the sensitivity lead to the second generation experiments such as ADMX (3) which is currently taking data with a bigger resonant 

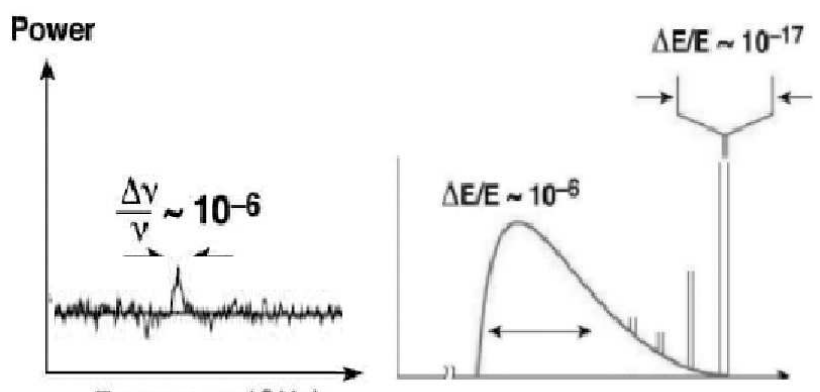

Frequency $(\mathrm{GHz})$

Fig. 2. Left: Representation of the signal expected in a microwave cavity experiment, where the photon signal appears as an excess over the noise in the power frequency spectrum. The width of the signal would be related with the predicted axion halo velocities. Right: Zoom showing the possible fine structure details in the expected signal

cavity as the precedent experiment, but still making use of the HFET amplifiers. Their results are compatible with the absence of any axion signal, providing the best upper limits for the axion to photon coupling constant $g_{a \gamma \gamma}$ in the lowest mass range $\left(m_{a} \approx 10^{-4}-10^{-6} \mathrm{eV}\right.$, see Fig. 26$)$. A new amplifier technology based on Superconducting Quantum Interference Devices (SQUID), currently under development, can further improve the sensitivity of ADMX, such that it is possible to probe the theoretically favoured axion parameter range ( see Chap. ?? and Fig. 26).

Another second generation experiment is the CARRACK experiment in Kyoto (4). They also use a resonant cavity to produce the axion-convertedphotons, but the signal detection is based on a Rydberg atom single-quantum detector, providing an extremely low-noise photon counting capability. This experiment is still in the development phase.

\section{Solar Axions Searches}

As seen from the Earth, the most important and strongest astrophysical source for axions is the core of the Sun. There, pseudoscalar particles like axions would be continuously produced in the fluctuating electric and magnetic fields of the plasma via their coupling to two photons. After production the axions would freely stream out of the Sun without any further interaction (given that the axion mean free path in the Sun is $\approx g_{10}^{-2} 6 \times 10^{24} \mathrm{~cm}$ for $4 \mathrm{keV}$ axions ${ }^{11}$, this is a valid assumption). The resulting differential solar axion flux on Earth would be (5)

$$
\frac{\mathrm{d} \Phi_{a}}{\mathrm{~d} E}=6.02 \times 10^{10} \mathrm{~cm}^{-2} \mathrm{~s}^{-1} \mathrm{keV}^{-1} g_{10}^{2} E^{2.481} e^{-E / 1.205} .
$$

${ }^{11}$ Here we define $g_{10}=g_{a \gamma \gamma} / 10^{-10} \mathrm{GeV}^{-1}$. 

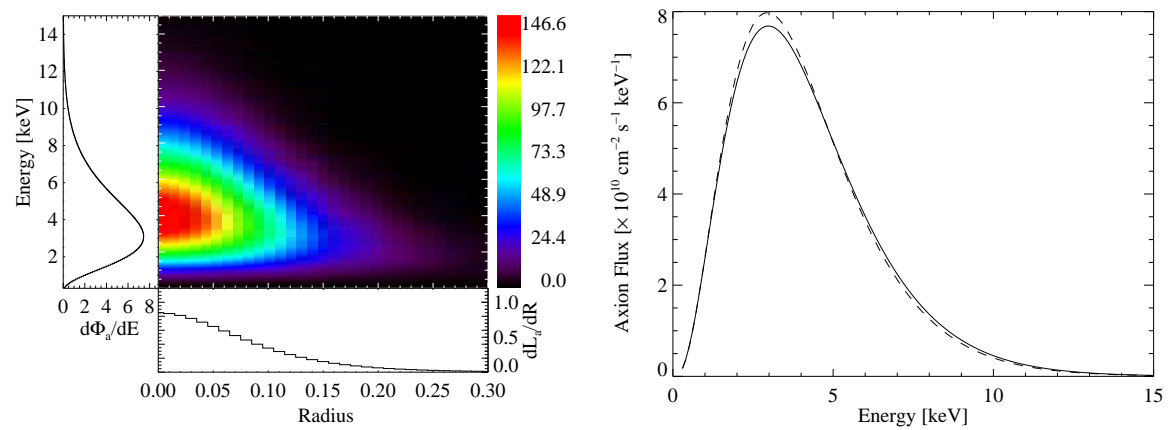

Fig. 3. Left: $2 \mathrm{D}$ representation of the axion surface luminosity seen from the Earth (5) as a function of the solar radius and the axion energy. Right: Comparison of the solar axion flux calculated using an older version of the standard solar model from 1982 (dashed line) and a more recent version from 2004 one (solid line) (6). Here an axion-photon coupling constant of $1 \times 10^{-10} \mathrm{GeV}^{-1}$ is assumed

The spectral energy distribution of the axions follows a thermal energy distribution between 1 and $10 \mathrm{keV}$, which peaks at $\approx 3 \mathrm{keV}$ as shown in the right part of Fig. 3. For comparison two different solar axion spectra are shown in Fig. 3, calculated from different versions of the standard solar model (SSM): the solid line corresponds to a more recent implementation of the SSM from 2004, while the spectrum plotted as a dashed line is based on an older version published in $1982(5 ; 6)$. It is evident that small changes of the underlying SSM have only a small influence on the axion flux and the corresponding shape of the axion energy spectrum. In the following we will summarize different techniques which aim to directly detect "solar axions".

\subsection{Axion Helioscope}

The most sensitive axion experiments at present in the mass range of $10^{-5} \mathrm{eV} \lesssim$ $m_{a} \lesssim 1 \mathrm{eV}$ are so called "axion helioscopes", i.e., magnetic solar telescopes. The underlying physical principle of such a telescope is based on the idea proposed by P. Sikivie in 1983 (7) and is illustrated in Fig. 4. If we assume that axions are produced in the Sun, they would reach the Earth after about $500 \mathrm{~s}$ as an almost parallel axion beam (the apparent diameter of the axion emission region on the solar disk is $\approx 0.1^{\circ}$ ). The apparatus for the detection on Earth uses the time reversed Primakoff effect, i.e., $a+\gamma_{\text {virtual }} \rightarrow \gamma$, where the axion interacts with a virtual photon provided by a transversal magnetic field and reconverts into a real photon. These "reconverted" photons have the same energy and momentum as the axion, and therefore their energy distribution is identical to the energy spectrum of the solar axions shown in Fig. 3. An X-ray detector placed on the end side of the magnetic field would collect the photons and search for an axion signature above detector background. The expected number of reconverted photons reaching the detector for the given 


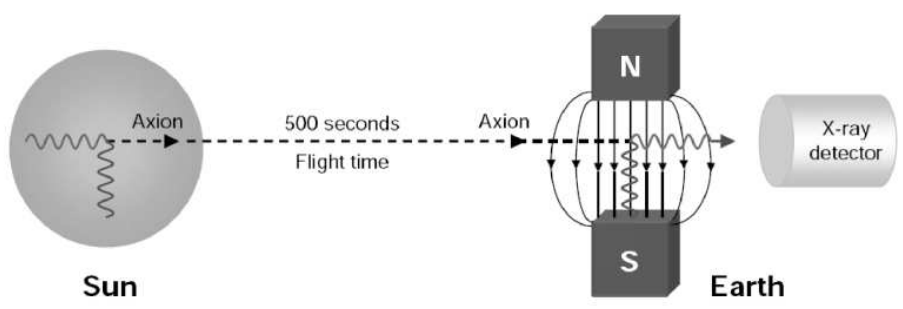

Fig. 4. Working principle of an axion helioscope. Axions produced in the Sun core by the Primakoff effect would be converted back into photons in a strong magnetic field. Eventually these photons, which have the same energy spectrum as the incoming axions, could be collected by a X-ray detector placed a the end of the magnet field area

differential solar axion spectrum is

$$
N=\int \frac{\mathrm{d} \Phi_{a}}{\mathrm{~d} E} P_{a \rightarrow \gamma} S t \mathrm{~d} E .
$$

Here $d \Phi_{a} / d E$ is the differential axion flux at the Earth, $S$ the magnet bore area, $t$ the measurement time, and $P_{a \rightarrow \gamma}$ the conversion probability of an axion into a photon. To maximize $t$ the magnet is mounted in a similar way as conventional telescopes, such that it can follow the track of the Sun on the sky.

The number of photons leaving the magnetic field towards the detector is determined by the probability that an axion converts back to a "observable" photon inside the magnetic field, assuming that the conversion volume (magnetic field area) is evacuated, the probability is given by

$$
P_{a \rightarrow \gamma}=\left(\frac{B g_{a \gamma \gamma}}{2}\right)^{2} 2 L^{2} \frac{1-\cos (q L)}{(q L)^{2}},
$$

where $B$ and $L$ are the magnet field strength and length of the field, and

$$
q=m_{a}^{2} / 2 E_{\gamma},
$$

is the longitudinal momentum difference (or momentum transfer) between the axion and the photon of the energy $E_{\gamma}$. In order to achieve a maximum conversion probability $P_{a \rightarrow \gamma}$ (constructive interference), the axion and photon fields must remain in phase over the length of the magnetic field, this sets the requirement that $q L<\pi(8 ; 10)$. Thus, the sensitivity of a helioscope is limited to a specific axion mass range for a given $q$. For increasing axion masses the conversion is suppressed by the momentum mismatch between the axion and the photon, namely due to the form factor $(1-\cos (q L)) /(q L)^{2}$ in (3).

Coherence can be restored for higher axion masses by filling the magnetic conversion region with a buffer gas (10), such that the photons inside the 
magnet pipe acquire an "effective mass" (which is equivalent to a refractive index $n$ ). Consequently the wavelength of the photon can match that of the axion for an appropriate gas pressure and coherence will be preserved for a narrow axion mass window. In this case the transition probability $P_{a \rightarrow \gamma}$ has to be recast into the more general equation (10)

$$
P_{a \rightarrow \gamma}=\left(\frac{B g_{a \gamma \gamma}}{2}\right)^{2} \frac{1}{q^{2}+\Gamma^{2} / 4}\left[1+e^{-\Gamma L}-2 e^{-\Gamma L / 2} \cos (q L)\right] .
$$

Here $\Gamma$ is the absorption coefficient for the X-rays in the medium and the momentum transfer becomes

$$
q=\left|\frac{m_{\gamma}^{2}-m_{a}^{2}}{2 E_{a}}\right|
$$

with the effective photon mass $m_{\gamma}$

$$
m_{\gamma} \simeq \sqrt{\frac{4 \pi \alpha n_{e}}{m_{e}}}=28.9 \sqrt{\frac{Z}{A} \varrho},
$$

where $n_{e}$ is the number density of electrons in the medium and $m_{e}$ the electron mass. The second expression in (7) is a more convenient representation for $m_{\gamma}$ giving the dependence of $m_{\gamma}$ on the atomic number $Z$ of the buffer gas, its mass $A$, and its density $\varrho$ in $\mathrm{g} \mathrm{cm}^{-3}$. Since Helium is the type of gas commonly used for this purpose (7) can be further simplified to

$$
m_{\gamma}(\mathrm{eV}) \simeq \sqrt{0.02 \frac{P(\mathrm{mbar})}{T(\mathrm{~K})}},
$$

when we use the corresponding parameters $A, Z$, and $\varrho$ for Helium and treat Helium as an ideal gas. This equation directly relates $m_{\gamma}$ to the measured experimental parameters, i.e., the pressure $P$ and temperature $T$ of the gas inside the magnetic field region. For a specific pressure $P$ and temperature $T$, the coherence is restored for a narrow axion mass window, for which the effective mass of the photon matches that of the axion such that

$$
q L<\pi \Longrightarrow \sqrt{m_{\gamma}^{2}-\frac{2 \pi E_{a}}{L}}<m_{a}<\sqrt{m_{\gamma}^{2}+\frac{2 \pi E_{a}}{L}} .
$$

The differential energy spectrum of the photons from axion conversion follows from (1) and (3). Assuming full coherence it can be approximated analytically by $(5)$

$$
\begin{aligned}
\frac{\mathrm{d} \Phi_{\gamma}}{\mathrm{d} E} & =\frac{\mathrm{d} \Phi_{a}}{\mathrm{~d} E} P_{a \rightarrow \gamma} \\
& =0.088 \mathrm{~cm}^{-2} \mathrm{day}^{-1} \mathrm{keV}^{-1} g_{10}^{4} E^{2.481} e^{-E / 1.205}\left(\frac{B L}{9.0 \mathrm{~T} 9.26 \mathrm{~m}}\right)^{2}
\end{aligned}
$$

It is obvious that the resulting low photon count rates from (10) require background optimized detectors in order to achieve a reasonable detection sensitivity with an axion helioscope. 


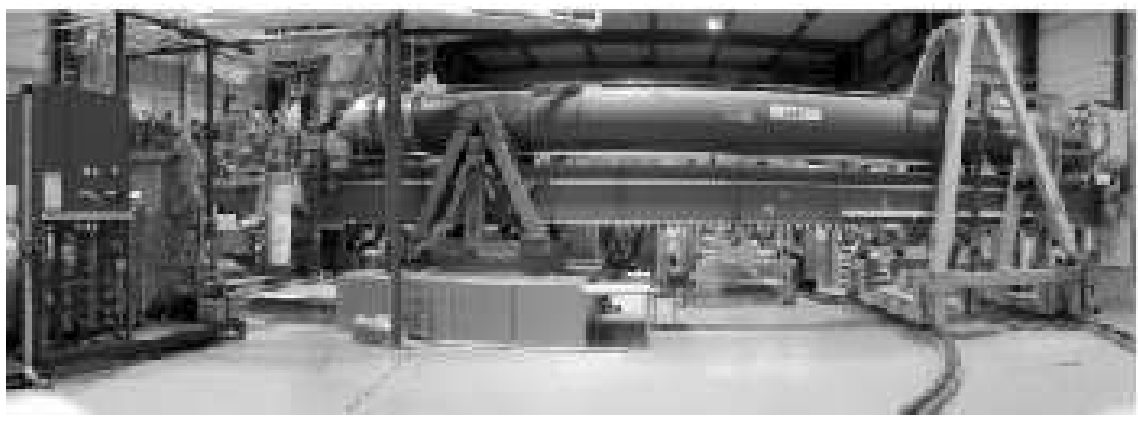

Fig. 5. Experimental setup of the CAST experiment at CERN. From the left to the right the Helium cryogenic system, the CAST superconducting magnet, and the tracking system is shown. The tracking system allows to follow the Sun $1.5 \mathrm{~h}$ per day during sunrise and sunset

\section{Helioscope Implementations}

The first axion helioscope was built at the beginning of the 90s (8), using a $180 \mathrm{~cm}$ long magnet powered with a $2.2 \mathrm{~T}$ transverse magnetic field. As a detector a proportional counter operating with a gas mixture of argon and methane $(90 \%$ to $10 \%)$ was used to collect the photons from axion to photon conversion. The helioscope could be pointed to the Sun for a period of $\approx$ 15 min day ${ }^{-1}$. Two different regions of the axion mass range (with and without a gas in the conversion volume) were explored with this setup, setting the following limits on $g_{a \gamma \gamma}$ and $m_{a}$ in the absence of a signal

$$
\begin{aligned}
& g_{a \gamma \gamma}<3.6 \times 10^{-9} \mathrm{GeV}^{-1} \text { for } m_{a}<0.03 \mathrm{eV}, \\
& g_{a \gamma \gamma}<7.7 \times 10^{-9} \mathrm{GeV}^{-1} \text { for } 0.03 \mathrm{eV}<m_{a}<0.11 \mathrm{eV} .
\end{aligned}
$$

In the late $90 \mathrm{~s}$ a new helioscope with improved sensitivity $(2.3 \mathrm{~m}$ long magnet, $4 \mathrm{~T}$ ) was built in Tokyo (11). For this experiment sixteen PIN photodiodes were used as X-ray detectors and the superconducting magnet was fixed on a polar mount, allowing the system to follow the Sun in a range from $-28^{\circ}$ to $+28^{\circ}$ in declination and $360^{\circ}$ in right ascension. Due to the better tracking system, the Tokyo helioscope was able to follow the Sun for $24 \mathrm{~h}$ a day and the observation time of the Sun could be significantly improved. In total the Tokyo collaboration was able to use about $50 \%$ of the duty cycle of their instrument to track the Sun, while the rest of the time was devoted to background measurements. Similar to the first approach of (8), the experiment was divided into two phases, the first phase was finished in 1997 with an evacuated conversion region and the second phase in 2000 when the magnet pipe was filled with helium gas in order to extend the sensitive axion mass range. The resulting upper limits on $g_{a \gamma \gamma}$ from both data taking periods are $(13 ; 12)$ 


$$
\begin{aligned}
& g_{a \gamma \gamma}<6 \times 10^{-10} \mathrm{GeV}^{-1} \text { for } m_{a}<0.03 \mathrm{eV}, \\
& g_{a \gamma \gamma}<6.8-10.9 \times 10^{-10} \mathrm{GeV}^{-1} \text { for } m_{a}<0.3 \mathrm{eV} .
\end{aligned}
$$

The so far most sensitive implementation of an axion helioscope is operating at present at CERN, the CERN Axion Solar Telescope (CAST). It was built in 2002 by refurbishing a $9.26 \mathrm{~m}$ long prototype of a twin aperture LHC dipole magnet. The two parallel pipes of the magnet, with a bore area of $A=14.5 \mathrm{~cm}^{2}$ each, are homogeneously interspersed by a transverse dipole magnetic field (see Fig. and 5 (14)). The maximum magnetic field that can be achieved with this prototype magnet is $\approx 9 \mathrm{~T}$. The magnet is mounted on an azimuthal moving platform that allows to track the Sun for $1.5 \mathrm{~h}\left( \pm 8^{\circ}\right.$ in height and $\approx 100^{\circ}$ in azimuth) during sunrise and sunset. To detect the axions, three different types of detectors are attached to the ends of the magnet pipes: a gaseous detector with a novel MICROMEGAS readout (15) and an X-ray telescope with a pn-CCD chip as focal plane detector (16) to detect axions during sunrise, and a Time Projection Chamber (TPC) which covers the opposite end of the magnet pipes, ready to collect axions during sunset (17).

CAST has taken data with the conversion region being evacuated during the years 2003 and 2004. In the absence of any axion signal, an upper limit on the axion to photon coupling constant has been established to $(5 ; 18)$

$$
g_{a \gamma \gamma}<0.88 \times 10^{-10} \mathrm{GeV}^{-1} \text { for } m_{a}<0.02 \mathrm{eV},
$$

at the $95 \%$ confidence level. This result is the best laboratory limit from a helioscope achieved so far. It covers a broad range of masses and supersedes the so far best limit from astrophysical considerations that was derived from the evolution of horizontal-branch stars in globular clusters $(19 ; 20)$. During 2005 the configuration of the CAST experiment was upgraded, ready to jump into the second phase of the experiment, when the magnet pipes are filled with ${ }^{4} \mathrm{He}$ or ${ }^{3} \mathrm{He}$ gas. In this configuration CAST will be able to expand its sensitivity to a maximum axion mass of $\approx 1.1 \mathrm{eV}$. CAST is scheduled to take data in this configuration during 2006 and 2007.

\subsection{Bragg Diffraction}

Another interesting and completely different approach to detect solar axions was proposed by E. A. Paschos and K. Zioutas (21). This detection principle uses the intense Coulomb field of nuclei in a crystal lattice instead of an external magnetic field to convert axions to photons by the Primakoff effect. A crystal can either be used simultaneously as an axion converter and detector or to build a "reflective parabolic antenna" covered with a thin film of crystals which focus the resulting photons from axion to photon conversion on a detector in their focal plane. Background optimized solid state detectors

(e.g., NaI or Ge) generally used for WIMP searches or in double beta decay 


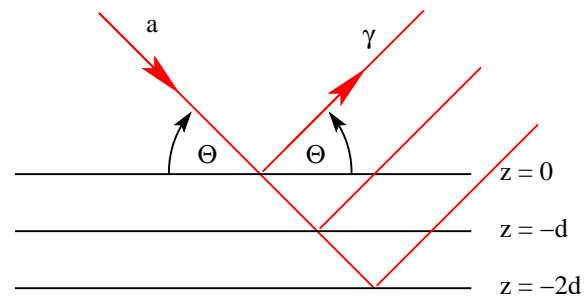

Fig. 6. Schematic view of different lattice layers in a crystal. The axion wave enters the crystal under the angle $\Theta$. If the Bragg condition is fulfilled the outgoing photon waves reflected from different lattice layers would interfere constructively and enhance the expected signal

experiments provide excellent performance to implement the first type of a crystalline axion detector.

To calculate the probability for axion conversion, the mass of the axion can be considered as negligibly small compared to the mass of the nuclei in the crystal, thus recoils of the nuclei can be neglected during the conversion process. In this case the energy of the outgoing photon would be equal the energy of the incident axion. The differential cross section for this conversion is given by $(21)$

$$
\frac{\mathrm{d} \sigma}{\mathrm{d} \Omega}=\frac{g_{a \gamma \gamma}}{16 \pi^{2}} F_{a}^{2}(\mathbf{q}) \sin ^{2}(2 \Theta) .
$$

Here $2 \Theta$ is the scattering angle and $F_{a}$ a form factor which describes the atomic structure of the crystal. The form factor $F_{a}$ can be calculated from the Fourier transformation of the electrostatic field $\Phi(\mathbf{x})$ in the crystal (22)

$$
F_{a}(\mathbf{q})=k^{2} \int \Phi(\mathbf{x}) e^{\mathrm{iq} \cdot \mathbf{x}} \mathrm{d}^{3} x
$$

with the transfered momentum $q=|\mathbf{q}|=2 k \sin (\Theta)$ and the axion momentum $k=|\mathbf{k}| \approx E_{a}$. Using the Sun as an axion source, the expected mean energy of the solar axions would be of the order of $\approx 4 \mathrm{keV}$ (see Fig. 3). Thus the wave length of the axion $\lambda$ is similar to the lattice spacing $d$ of the crystal $(\approx$ few $\AA)$, consequently we would expect a Bragg-reflection pattern. If a Bragg pattern arises, the intensity of the X-rays leaving the crystal has a maximum if the Bragg-condition

$$
2 d \sin \left(\Theta_{\text {Bragg }}\right)\left(1-\frac{1-n}{\sin ^{2}\left(\Theta_{\text {Bragg }}\right)}\right)=m \lambda \quad \text { with } \quad m=1,2,3, \ldots,
$$

is fulfilled. Here we use $n$ for the refractive index and $d$ for the lattice spacing (compare Fig. 6). From (16) it is obvious that constructive interference will occur only if the waves reflected by different lattice layers are in phase. This is equivalent to the requirement that the path length of each photon wave leaving the crystal has to be equal to an integer multiple of its wavelength. 
The constructive interference then leads to an enhancement of the expected signal of the order of $10^{4}$ compared to a scattering off a single atom in the crystal (21).

For a detector with a fixed orientation of its crystalline lattice relative to the Earth's surface the angle $\Theta$, which is basically the angle between the line of sight towards the Sun and the crystalline lattice, would change during the course of the day when the Sun moves across the sky. Hence, the expected photon signal would have a well defined temporal signature since the Bragg condition is only satisfied for specific times during the day. This signature makes a potential signal clearly distinguishable from the detector background.

It was shown by (22) that the maximum achievable sensitivity for an upper limit on $g_{a \gamma \gamma}$ with this kind of detection technique is of the order of $g_{a \gamma \gamma} \lesssim 1 \times 10^{-9} \mathrm{GeV}^{-1}$ using a $100 \mathrm{~kg}$ DAMA like NaI detector (23), assuming an observation time of 2 years, and a detector background of 2 counts kg ${ }^{-1}$ day $^{-1} \mathrm{keV}^{-1}$. It is obvious from this estimate, that axion detection with crystals based on Bragg reflection is not competitive to, e.g., axion helioscopes or astrophysical observations in terms of sensitivity with respect to $g_{a \gamma \gamma}$. An alternative and more effective detector configuration would be a large surface parabolic reflector ("mirror") or an array of reflectors covered with a thin crystalline layer (21). In addition polar crystals (ferroelectric) materials can improve the sensitivity, since they provide stronger microscopic electric fields (21). Nevertheless, the Bragg reflection technique has a major advantage, its sensitivity does not dependent on the mass of the axion, as long as nuclear recoils can be neglected.

Two different collaborations, COSME (24) and SOLAX (25), both using Germanium detectors with the main purpose of searching for Dark Matter WIMPs, analyzed their data looking for an axion signature. Both experiments were able to yield very similar mass-independent bounds to the axion to photon coupling in the absence of any signal

$$
\begin{array}{ll}
g_{a \gamma \gamma}<2.7 \times 10^{-9} \mathrm{GeV}^{-1} & \text { (SOLAX) }, \\
g_{a \gamma \gamma}<2.8 \times 10^{-9} \mathrm{GeV}^{-1} & \text { (COSME). }
\end{array}
$$

The DAMA collaboration (26) achieved a similar limit

$$
g_{a \gamma \gamma}<1.7 \times 10^{-9} \mathrm{GeV}^{-1} \quad(\mathrm{DAMA}),
$$

using $\mathrm{NaI}(\mathrm{Tl})$ crystals as detector material.

\subsection{Geomagnetic Axion Conversion}

It has recently been shown by (28) that Geomagnetic Conversion of Solar Axions to X-rays (GECOSAX) can yield a photon flux which is measurable by a satellite based X-ray observatory on the dark side of the Earth. A key ingredient of this idea is to use the Earth as an X-ray shield and look for axions 

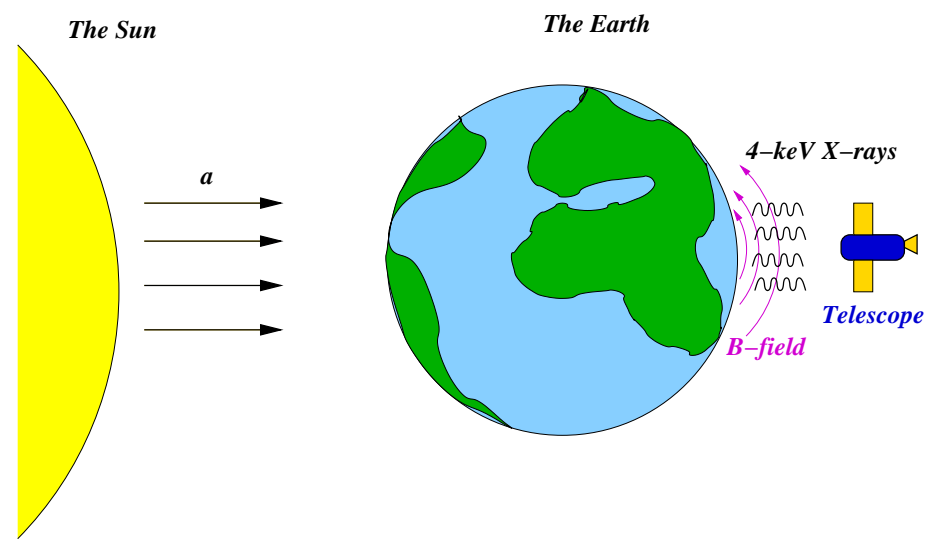

Fig. 7. The observational setup to detect geomagnetic converted axions (GECOSAX). The Sun is used as an axion source and a satellite based X-ray observatory to detect the photons from geomagnetically converted axions. The Earth acts as a shield for direct X-rays coming from the Sun and other X-ray sources on the sky

on its dark side. Using the Earth as a "shield" towards the Sun, effectively removes the solar X-ray background (see Fig. 7 for a schematic representation of this setup). In the following section, the feasibility of such an experiment will be demonstrated, based on recent measurements of the dark-Earth X-ray background by the Suzaku satellite.

The radius of the Earth is $R_{\oplus} \approx 6.4 \times 10^{3} \mathrm{~km}$ and its magnetic field is well approximated by a dipole for distances less than $1000 \mathrm{~km}$ above the surface. The magnetic field strength at the equator is $B_{\oplus} \simeq 3 \times 10^{-5} \mathrm{~T}$ and it drops as $\propto 1 / r^{3}$ (58). However, over distances $L \ll R_{\oplus}$ above the Earth's surface, it can be assumed that $B_{\oplus}=$ const. As we are interested in low Earth orbits $(L<1000 \mathrm{~km})$, this is a valid assumption (28). The influence of the atmosphere of the Earth is negligible, since for $L>150 \mathrm{~km}$ solar axions essentially travel in vacuum. For an axion mass $m_{a} \leq 10^{-4} \mathrm{eV}$, a mean axion energy $E_{a}=4 \mathrm{keV}$, and a satellite orbit of $L_{\oplus} \simeq 600 \mathrm{~km}$, the axion to photon conversion probability can be approximated as

$$
P_{a \rightarrow \gamma}(L)=\frac{1}{4}\left(g_{a \gamma \gamma} B L\right)^{2}
$$

where $g_{a \gamma \gamma}$ is the coupling strength of the axion to the photon. To be able to compare the expected axion flux in a specific energy range with available data, the spectrum given by (1) is integrated over the energy range of 2 to $7 \mathrm{keV}$ to find the solar axion flux at Earth to be

$$
\Phi_{a} \approx 2.7 \times 10^{11}\left(g_{a \gamma} / 10^{-10} \mathrm{GeV}^{-1}\right)^{2} \mathrm{~cm}^{-2} \mathrm{~s}^{-1} .
$$

Thus, using (19) the expected flux of X-rays from axion to photon conversion at an altitude of $L_{\oplus}$ near the equator for $g_{a \gamma}=10^{-10} \mathrm{GeV}^{-1}$ and $B=B_{\oplus}$ is 


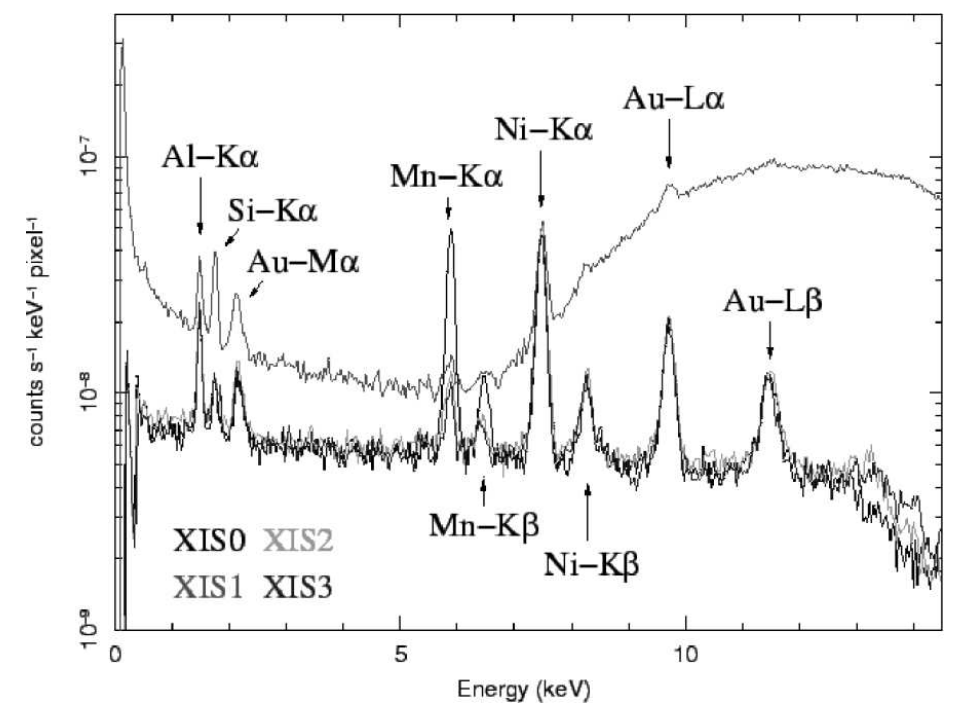

Fig. 8. Dark-Earth background measured by Suzaku based on a $800 \mathrm{ksec}$ long observation. Individual fluorescent emission lines originating in detector materials are marked (30)

$$
\Phi_{\gamma}\left(L_{\oplus}\right) \equiv \Phi_{a} P_{a \rightarrow \gamma}\left(L_{\oplus}\right) \approx 3 \times 10^{-7} \mathrm{~cm}^{-2} \mathrm{~s}^{-1}
$$

Based on the published technical specifications (27) of the Rossi X-ray timing explorer (RXTE), (28) have estimated that the flux given by (21) can be detected on the dark side of the Earth.

However, recently the Suzaku X-ray satellite team has measured the darkEarth X-ray background in the range of 2 to $7 \mathrm{keV}$ for calibration purposes (29). Suzaku has a circular orbit at $575 \mathrm{~km}$ with an inclination of $33^{\circ}$. Unfortunately, the Suzaku satellite does not provide a fast slew rate and thus cannot perform this kind of measurement. However, as will be shown below, a telescope, capable of tracking the solar core on the dark side of the Earth and with similar X-ray detection capabilities will be sensitive to axion-photon couplings $g_{a \gamma \gamma}$ far below the current existing laboratory and astrophysical bounds for axion masses $m_{a}<10^{-4} \mathrm{eV}$.

The expected signal from axion to photon conversion has several distinct features which will allow to clearly distinguish it from the detector background. These features are

1. a thermal like spectral distribution of X-rays peaked at approximately $4 \mathrm{keV}$, on the night side of the Earth,

2. the X-rays would only come from the center of the Sun, which subtends approximately $3^{\prime}$ on the sky,

3. the observed X-ray intensity would vary with the magnetic field strength and thus with the orbital position of the satellite and its orientation rel- 


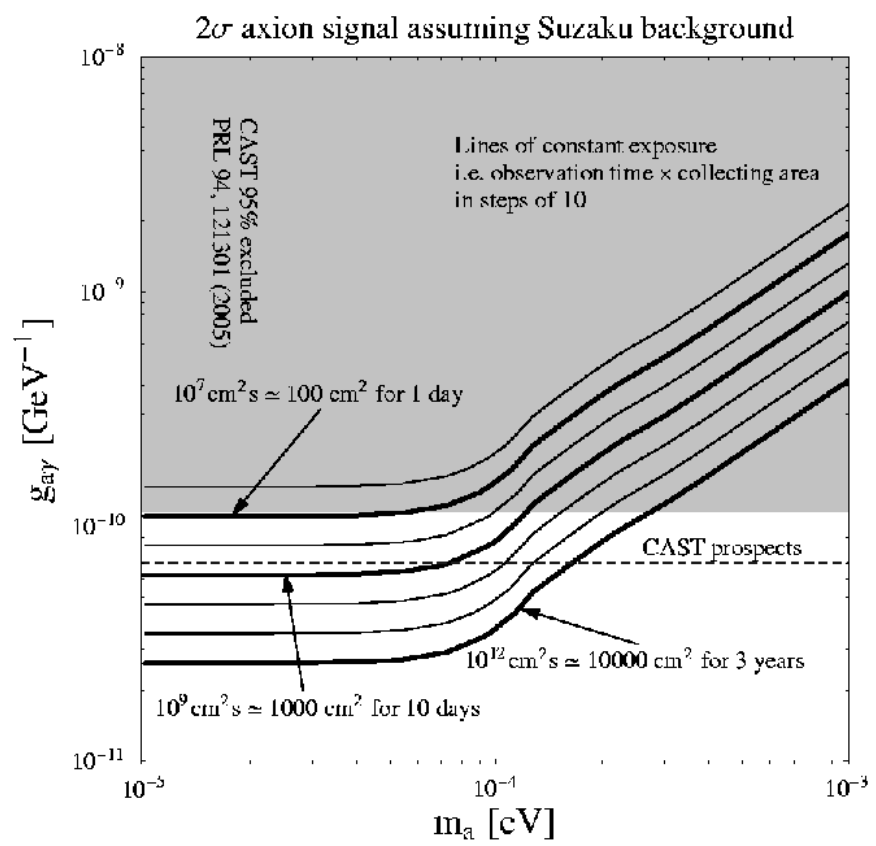

Fig. 9. The shaded area schematically depicts the CAST 95\% C.L. excluded region (5). The lines show sensitivities obtainable with the background measured by Suzaku

ative to the Earth's magnetic field (pointing direction of the satellite), and

4. the signal would be modulated with the Sun-Earth distance, resulting in an annual modulation of the intensity of the signal.

To estimate the sensitivity of Suzaku for solar axion detection, the effective area of the Suzaku detector was assumed to be $\approx 300 \mathrm{~cm}^{2}$ in the $2-7 \mathrm{keV}$ range (29). The expected dark Earth background of the Suzaku XIS detector per unit area is presented in Fig. 8 (30). Given the above effective area, the $\approx 1^{\prime}$ resolution of the X-ray telescope and the Suzaku dark Earth background data, the measurement of the X-ray flux from solar axion conversion is clearly feasible. The Suzaku team estimates that with $3 \times 10^{5} \mathrm{~s}$ of data, a $4 \sigma$ bound $g_{a \gamma \gamma}<10^{-10} \mathrm{GeV}^{-1}$ would have been possible (29). Based on the Suzaku background data (29), the sensitivity of a solar-core-tracking X-ray telescope is plotted as a function of $m_{a}$ in Fig. 9. The Earth-occulted background can in general be measured in situ, by pointing the X-ray telescope away from the direction of the core of the Sun. The observation of such a signal amounts to viewing an X-ray image of the solar core through the Earth. Therefore, this method can achieve an unambiguous detection of solar axions. In summary, for solar axions with $m_{a}<10^{-4} \mathrm{eV}$, an orbiting X-ray telescope, with an 
effective area of a few $10^{3} \mathrm{~cm}^{2}$, can probe solar axion $g_{a \gamma \gamma}$ well beyond the sensitivity of current laboratory experiments.

\section{Searches for Laser Induced Axions}

In this section, a new generation of purely laboratory based experiments which are able to provide complementary results to solar axions searches, will be briefly introduced. Whereas the solar axion experiments, described in the previous sections, probe axions which would escape from the Sun, the experiments presented in the following are supposed to produce axions from polarized laser beams, propagating in a transverse magnetic field. Yet the mechanism for both, the production and detection, is the same as in the previously described experiments: the Primakoff effect.

Searches for laser induced axions provide a fully model independent approach, since they do not rely on the physical processes and conditions in the Sun and how axions or axion-like particles could be produced under these conditions. They can be viewed as fixed target type experiments, with low energy polarized photon beams colliding with virtual photons provided by the magnetic field, i.e., $\gamma+\gamma_{\text {virtual }} \rightarrow a$. By pushing the optical detection techniques up to the present state-of-art, a significant enlarged domain of axion mass and axion di-photon coupling constant, not yet explored with laser based experiments, is expected to be probed with photons, typically in the energy range of a few $\mathrm{eV}$. In addition, laser based experiments are not only focused on the axion search, but offer also a broad band of scientific interests, starting from a precise new test of QED up to the search of any scalar, pseudoscalar, or other particles like paraphotons or millicharged particles that can couple to photons. In general, laser based experiments can be divided into two categories,

- the so called "photon regeneration" or "light shining through a wall" experiments and

- experiments that probe the magneto-optical properties of the vacuum.

\subsection{Light Shining Through a Wall}

The light shining through a wall or photon regeneration principle was first proposed by (31) in 1987 (for an independent proposal of this type, see (9)). The basic idea of this experiment is shown in Fig. 10. A polarized laser beam propagates inside a transverse magnetic field (with $\mathbf{E} \| \mathbf{B}$ ). The laser is blocked by a wall at some point of its path, such that only weakly interacting pseudoscalar or scalar particles created before will be able to pass through this absorber. The conversion probability for the production of an axion-like particle in this case is given by

$$
P_{\gamma \rightarrow a} \propto \frac{1}{4}\left(g_{a \gamma \gamma} B L\right)^{2} F(q L),
$$




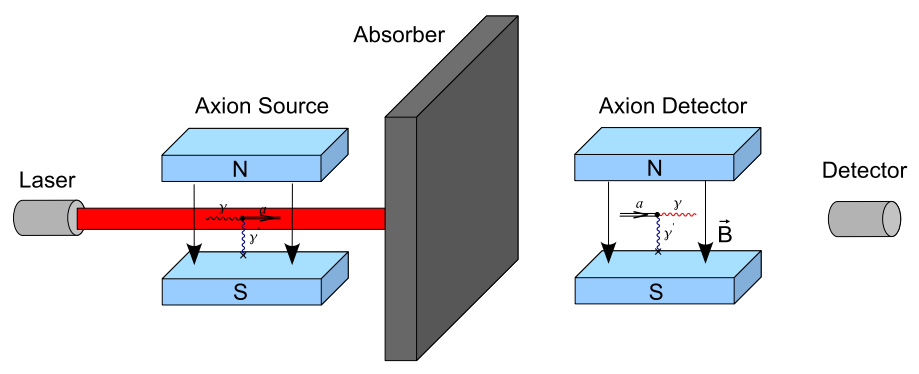

Fig. 10. Schematic view of a photon regeneration or light shining through a wall experiment. A polarized laser beam enters a transverse magnetic field from the left. A small fraction of the laser photons can be transmuted to, e.g., axions. While the laser light is blocked by a thick absorbing wall, the axions would pass it. A second magnetic field behind the wall is used to reconvert the axions to photons which are finally detected (31)

were $F(q L)$ is the form factor given by (3). A fraction of these weakly interacting particles will turn back into detectable photons after they have passed the wall and enter the second magnetic field, called "Axion Detector" in Fig. 10. The total probability to observe a regenerated photon with a detector located at the end of the second magnetic field is

$$
P_{\gamma \rightarrow a \rightarrow \gamma}=P_{\gamma \rightarrow a}^{2} .
$$

The expected counting rate depends on the power of the laser $P$ and can be enhanced by an optical cavity installed on the production side. If the total number of reflections in the primary magnet is $N_{\mathrm{r}}$ and the detection efficiency is $\eta$, then the total number of counts expected from reconversion calculates from (32)

$$
\begin{aligned}
\frac{\mathrm{d} N_{\gamma}}{\mathrm{d} t} & =\frac{1}{16} \frac{\langle P\rangle}{\omega} \frac{N_{\mathrm{r}}+2}{2} \eta\left(g_{a \gamma \gamma} B L\right)^{4} \sin ^{2}\left(\frac{m_{a}^{2} L}{4 \omega}\right) /\left(\frac{m_{a}^{2} L}{4 \omega}\right)^{4} \\
& \approx \frac{1}{16} \frac{\langle P\rangle}{\omega} \frac{N_{\mathrm{r}}+2}{2} \eta\left(g_{a \gamma \gamma} B L\right)^{4},
\end{aligned}
$$

where the approximation (25) is valid only for $m_{a} \ll \sqrt{2 \pi \omega / L}$.

The pioneering experiment based on this technique was performed by the Brookhaven-Fermilab-Rutherford-Trieste (BFRT) collaboration. They used two superconducting dipole magnets of length $L=4.4 \mathrm{~m}$ which were able to provide a magnetic field of $B=3.7 \mathrm{~T}$. The optical laser with a wavelength of $\lambda=514 \mathrm{~nm}$ had an average power of $\langle P\rangle=3 \mathrm{~W}$ and was operated in an optical cavity providing 200 reflections. Since no signal from photon regeneration was found, the BFRT collaboration was able to set an upper limit on $g_{a \gamma \gamma}$ of (35)

$$
g_{a \gamma \gamma}<6.7 \times 10^{-7} \mathrm{GeV}(95 \% \text { C.L. }) \text {, }
$$



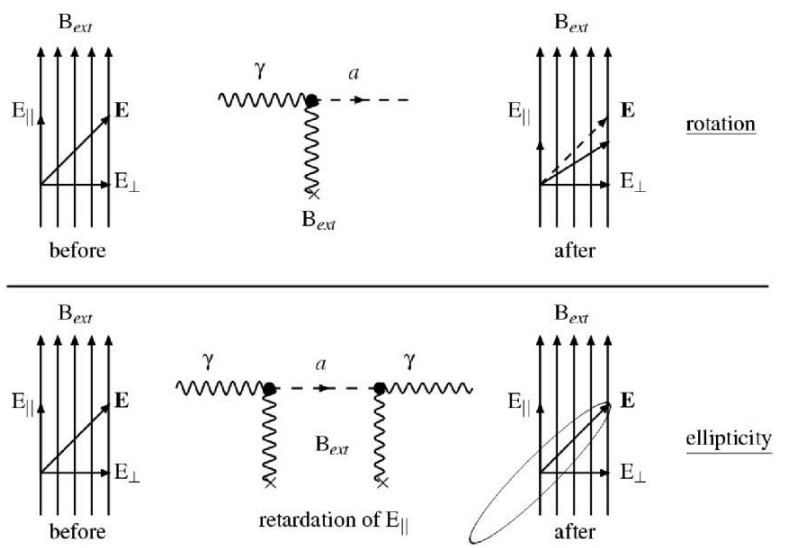

Fig. 11. Up: Linear Dichroism or rotation of the polarization vector by and angle $\varepsilon$. Down: Linear Birefringence or induction of an ellipticity $\Psi$ in an initially linear polarized beam

for axion-like pseudoscalars with a maximum mass of

$$
m_{a}<10^{-3} \mathrm{eV} .
$$

\subsection{Probing the Magneto-Optical Properties of the Vacuum}

The second of the purely laboratory experimental approaches to search for axions and axion-like particles is of indirect detection type. It is based on the theoretical prediction that scalar or pseudo-scalar particles can affect the polarization of light propagating in vacuum through a transverse magnetic field $\mathbf{B}$ because of their coupling to photons (43) (see also Chap. ??). A light beam initially linearly polarized with an angle $\theta$ with respect to $\mathbf{B}$ is expected to acquire a small ellipticity $\Psi$ and a small apparent rotation $\Theta$ due to dispersive and absorptive processes induced by the production of spinless particles. Both effects are usually referred to as birefringence and as linear dichroism of the vacuum and are characterized by

$$
\begin{gathered}
\Psi \approx N \frac{B^{2} L^{3} m_{A}^{2}}{96 \omega M^{2}} \sin (2 \theta), \\
\Theta \approx N \frac{B^{2} L^{2}}{16 M^{2}} \sin (2 \theta),
\end{gathered}
$$

in the limit $m_{A}^{2} L / 4 \omega \ll 1$. Here $m_{A}$ is the axion mass, $M=1 / g_{a \gamma \gamma}$ the inverse coupling constant to two photons, $\omega$ the photon energy, $L$ and $N$ the effective path lengths and the number of paths the light travels in the 
transverse magnetic field. The polarization state of the light beam leaving the magnetic field, characterized by (28) and (29), would manifest itself as a sizeable deviation from the pure QED prediction $(44 ; 45)$ for which no measurable linear dichroism is expected.

The photon splitting effect can also produce a differential absorption (46), giving rise to an apparent angular rotation of the polarization of the order of $10^{-34} \mathrm{rad}$ in a $9.5 \mathrm{~T}$ magnetic field over a length of $25 \mathrm{~km}$. This angular rotation angle is far from being measurable under laboratory conditions, except if the coupling with scalar or pseudo-scalar particles would significantly enhance this effect (47).

The vacuum magnetic birefringence predicted by the QED, corresponds to the dispersive effect produced by virtual electron-positron pairs as this was already stated by Heisenberg and Euler in 1936 (44): "Even if electromagnetic fields are not strong enough to create matter, they will, due to the virtual possibility of creating matter, polarize the vacuum and therefore change the Maxwell's equations". This ellipticity constitutes the background signal for a measurement that aims to detect a birefringence or dichroism induced by a spinless particle. The ellipticity expected from vacuum-polarization can be expressed from one loop calculation as $(44 ; 45)$

$$
\Psi_{\mathrm{QED}} \approx N \frac{B^{2} L \alpha^{2} \omega}{15 \mathrm{~m}^{4}} \sin (2 \theta)
$$

where $\alpha \approx 1 / 137$ is the fine-structure constant, $\omega$ is the photon energy and $m_{e}$ the electron mass. The maximum of ellipticity, a laser beam with a wavelength $\lambda=1550 \mathrm{~nm}$ propagating in a $9.5 \mathrm{~T}$ field over a length $N L=25 \mathrm{~km}$ would acquire is equal to $2 \times 10^{-11} \mathrm{rad}$.

A search for both effects, birefringence and dichroism, was carried out with the same magnets used in the BFRT experiment (35), setting a bound on the axion to photon coupling constant of

$$
g_{a \gamma \gamma}<3.6 \times 10^{-7} \mathrm{GeV}^{-1},
$$

for masses

$$
m_{a}<5 \times 10^{-4} \mathrm{eV}
$$

at the $95 \%$ C.L.

Very recently the Italian PVLAS experiment (33) (see Chap. ??) has been taking data to test the vacuum birefringence in the presence of a magnetic field with a $1 \mathrm{~m}$ long dipole magnet operated at a maximum field of $5.5 \mathrm{~T}$, therefore improving the sensitivity of the previous experimental setups. For the first time the PVLAS collaboration has measured a positive value for the amplitude of the rotation $\varepsilon$ of the polarization plane in vacuum with $B \approx 5 \mathrm{~T}$ (quoted with a $3 \sigma$ uncertainty interval) (33)

$$
\varepsilon=(3.9 \pm 0.5) \times 10^{-12} \mathrm{rad} \mathrm{pass}^{-1} .
$$


Table 1. Planned laser based experiments to detect axion-like particles. The most important magnet and laser parameters are shown. PVLAS, and later also OSQAR, will use optical cavities to enhance the number of generated ALPs. $P_{\gamma \Phi \gamma}$ (PVLAS) denotes the probability for a photon-ALP-photon conversion for the different experimental setups based on the coupling constant derived from the PVLAS measurement and the BFRT limits. The last column gives the expected signal rate of re-converted photons

\begin{tabular}{|c|c|c|c|c|c|}
\hline Name & Location & Laser & Magnet & $\begin{array}{c}P_{\gamma \phi \gamma} \\
(\mathrm{PVLAS})\end{array}$ & $\gamma$ Flux \\
\hline ALPS & DESY/D & $\begin{array}{l}\lambda=1064 \mathrm{~nm} \\
P=200 \mathrm{~W}\end{array}$ & $\begin{array}{l}B=5 \mathrm{~T} \\
L=4.21 \mathrm{~m}\end{array}$ & $\sim 10^{-19}$ & $10^{1} \mathrm{~s}^{-1}$ \\
\hline BMV & LULI/F & $\begin{array}{l}\lambda=1053 \mathrm{~nm} \\
P=500 \mathrm{~W}\end{array}$ & $\begin{array}{l}B=11 \mathrm{~T} \\
L=0.25 \mathrm{~m}\end{array}$ & $\sim 10^{-21}$ & 10 pulse $^{-1}$ \\
\hline LIPSS & $\mathrm{Jlab} / \mathrm{USA}$ & $\begin{array}{l}\lambda=900 \mathrm{~nm} \\
P=10 \mathrm{~kW}\end{array}$ & $\begin{array}{l}B=1.7 \mathrm{~T} \\
L=1 \mathrm{~m}\end{array}$ & $\sim 10^{-23.5}$ & $10^{-1} \mathrm{~s}^{-1}$ \\
\hline OSQAR & $\mathrm{CERN} / \mathrm{CH}$ & $\begin{array}{l}\lambda=540 \mathrm{~nm} \\
P=1 \mathrm{~kW}\end{array}$ & $\begin{array}{l}B=9.5 \mathrm{~T} \\
L_{1}=1 \mathrm{~m} \\
L_{2}=13.3 \mathrm{~m}\end{array}$ & $\sim 10^{-18}$ & $10^{3} \mathrm{~s}^{-1}$ \\
\hline PVLAS & Legnaro/I & $\begin{array}{l}\lambda=1064 \mathrm{~nm} \\
P=0.8 \mathrm{~W}\end{array}$ & $\begin{array}{l}B_{1}=5 \mathrm{~T} \\
B_{2}=2.2 \mathrm{~T} \\
L_{1}=4.21 \mathrm{~m} \\
L_{2}=0.5 \mathrm{~m}\end{array}$ & $\sim 10^{-23}$ & $10^{-1} \mathrm{~s}^{-1}$ \\
\hline
\end{tabular}

This signal can be translated to an allowed range for the mass $m_{b}$ and the coupling constant to two photons $g_{b \gamma \gamma}$ of a neutral light pseudoscalar boson

$$
\begin{aligned}
1 \mathrm{meV} & \leq m_{b} \leq 1.5 \mathrm{meV} \\
1.7 \times 10^{-6} \mathrm{GeV}^{-1} & \leq g_{b \gamma \gamma} \leq 1 \times 10^{-5} \mathrm{GeV}^{-1}
\end{aligned}
$$

Several new experiments are planned and already in progress with the goal to verify this signal. They are based on both experimental techniques: the light shining through a wall principle and the principle to test the properties of the quantum vacuum or a combination of both. In Table 1, a summary of actually planned or already operating experiments is given together with their performance parameters. A more detailed introduction to some of the experiments quoted in Table 1 is given in the following sections.

\section{Axion-Like Particle Search - ALPS}

A collaboration of DESY, Laser Zentrum Hannover and Sternwarte Bergedorf is presently setting up the Axion-Like Particle Search (ALPS) photon regeneration experiment which exploits the photon beam of high-power optical lasers, sent along the transverse magnetic field of a superconducting HERA dipole magnet (34). This experiment, which has been approved by the DESY directorate on January 11, 2007, and is expected to take data in summer 2007, 


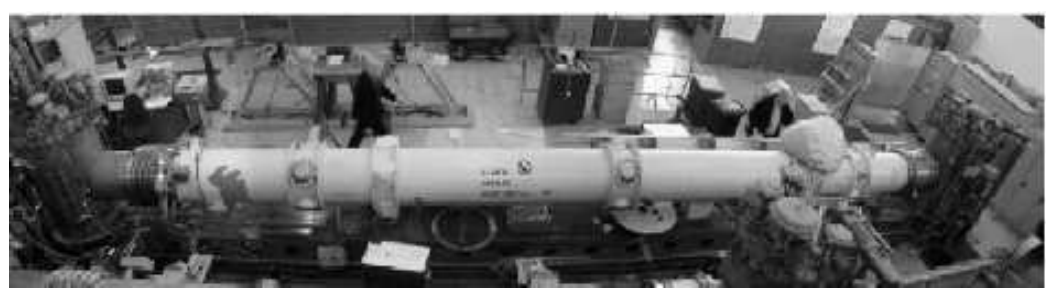

Fig. 12. Superconducting HERA dipole magnet exploited by ALPS for conversion of laser photons into axion-like particles, as well as for the reconversion of axion-like particles into photons

offers a window of opportunity for a rapid firm establishment or exclusion of the axion-like particle interpretation of the anomaly published by PVLAS. In case of confirmation, it would also allow for the measurement of mass, parity, and coupling strength of this particle.

The photon regeneration experiment ALPS is build around a spare dipole of the HERA proton storage ring at the DESY magnet test stand (see Fig. 12). Both parts of the experiment, i.e., axion-like particle production and reconversion into photons are accommodated in one single magnet, since the test stand architecture in its present configuration forbids to place two fully functional magnets in line. The general layout of the experiment is depicted in Fig. 13. A high intensity laser beam is placed on one side of the magnet traversing half of its length. In the middle of the magnet, the laser beam is reflected back to its entering side, and an optical barrier prevents any photons from reaching the second half of the magnet. Axion-like particles would penetrate the barrier, eventually reconverting into photons inside the second half of the magnet. Reconverted photons are then detected with a pixeled semi-conductor detector outside the magnet.

The magnetic field will be produced by a spare dipole magnet of the HERA proton storage ring (see Fig. 12). At a nominal current of $6000 \mathrm{~A}$, the magnet reaches a field of $5.4 \mathrm{~T}$ over a total magnetic length of $8.82 \mathrm{~m}$. In a first stage, ALPS will exploit a laser system delivering a linearly polarized photon beam with an average power of $50 \mathrm{~W}$ at a wavelength of $532 \mathrm{~nm}$ and a low noise, high quantum efficiency commercial CCD camera. Already with this configuration, the axion-like particle interpretation of PVLAS can be clarified: in this case, the expected counting rate is about $2 \mathrm{~Hz}$. In a second stage, it is planned to use a commercial fiber laser (Nd:YAG) at $1064 \mathrm{~nm}$ which is able to deliver $200 \mathrm{~W}$ linearly polarized photons, together with a InGaAs pixel detector normally used in infrared astronomy. This configuration will enable ALPS to explore also "new territory" in the coupling vs. mass plot. 


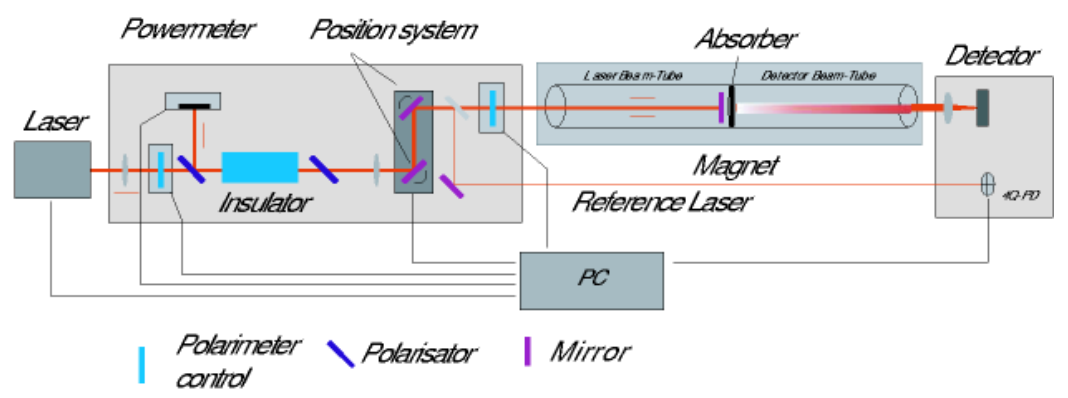

Fig. 13. Schematic view of the experimental setup with the laser on the left, followed by the laser injector/extractor system, the magnet and the detector table. An intensity-reduced reference beam of the laser is guided parallel to the magnet for constant alignment monitoring between laser and detector

\section{The BMV Project}

The BMV (Birefringence Magnetique du Vide) project (42), which is being built in Toulouse, France, combines very intense pulsed magnetic fields, developed at LNCMP, and a very sensitive optical device to detect the effects induced on a laser beam by such fields. This device is developed at LCARIRSAMC in collaboration with LMA-VIRGO from IN2P3 in Lyon, France. The goal was to assemble a first version of the experiment in 2006, and to obtain first results in 2007. In particular, the setup will be able to test the results published by the PVLAS collaboration (33).

The BMV project is based on a Fabry-Pérot cavity with a finesse of about $1 \times 10^{6}$ and a pulsed magnetic field as high as $25 \mathrm{~T}$ over the length of about one meter. The sensitivity limit for the measured ellipticity is about $4 \times 10^{-9}$, more than 100 times the one expected with the PVLAS apparatus. In the final stage, the experiment will have the sensitivity to measure the vacuum birefringence as predicted by QED.

The experimental setup of the optical apparatus is shown in Fig. 16. The light provided by a Nd:YAG laser $(\lambda=1064 \mathrm{~nm})$ laser is polarized and it is injected in a Fabry-Pérot cavity containing the magnetic field region. The light exiting the cavity is analyzed by a polarizer and detected by a photodiode. Light reflected back by the cavity is collected and this signal is used to drive the locking electronics that changes the laser frequency to keep the FabryPérot resonating. The measurement will be performed by phase detection, since the magnetic field is pulsed.

To achieve a maximum in sensitivity, the project demands a transverse magnetic field as high as possible. This can only be obtained using pulsed magnet techniques. Moreover, a magnetic field region as long as possible is required, since the QED effect depends on the product $B^{2} L$, where $L$ is the length of the magnetic field region. The goal is to realize a pulsed magnet 


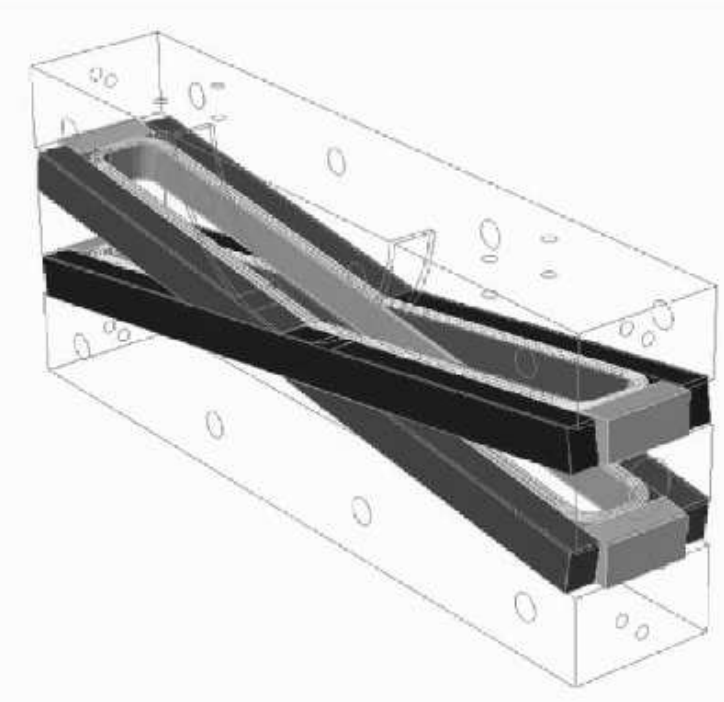

Fig. 14. Schematic view of the X-coil used in BMV

delivering a transverse magnetic field approaching $25 \mathrm{~T}$. Due to the high field value the structure of the magnet is very constraint. In particular, at this field level, the magnetic pressure corresponds to $250 \mathrm{MPa}\left(2.5 \mathrm{t} \mathrm{cm}^{-2}\right)$. Pulsed magnets that can provide such high fields have already been developed and tested, based on the X-coil geometry shown in Fig. 14. The length of the coil is $0.25 \mathrm{~m}$, providing a peak field of $14.3 \mathrm{~T}$ corresponding to about $28 \mathrm{~T}^{2} \mathrm{~m}$ (see Fig. 15). This is one of the highest field strengths ever reached with such a configuration. One of the prototype coils has been aged at a duty cycle of about 5 pulses per hour. It has delivered 100 pulses at a peak field of $11.5 \mathrm{~T}$ and 100 pulses at a peak field of $12.5 \mathrm{~T}$, corresponding to about $21 \mathrm{~T}^{2} \mathrm{~m}$. The magnet will be supplied by a pulsed generator based on a bank of 12 capacitors. In particular, this generator will be able to produce a single pulse or to give a field that oscillates.

As far as the optical system is concerned, a system of high precision mechanical translators and rotators for the Fabry-Pérot cavity mirrors and for the polarizers has been designed and assembled at LCAR in Toulouse. The piezoelectric stacks of the mirrors orientation system, as well as the mechanism allowing their rotation are adapted to ultra-high vacuum. Tests have been carried out with a vertical $3.6 \mathrm{~m}$ long Fabry-Pérot cavity based on the LMA mirrors, made to reach a finesse around $5 \times 10^{5}$. Figure 16 shows a schematic drawing of the experiment with the Fabry-Pérot resonator, the magnetic field region, and two cryostat. The chambers for the optics operated at a ultra high vacuum, are fixed on an optical table with a length of $3.6 \mathrm{~m}$. The length of the cavity is about $2 \mathrm{~m}$. 


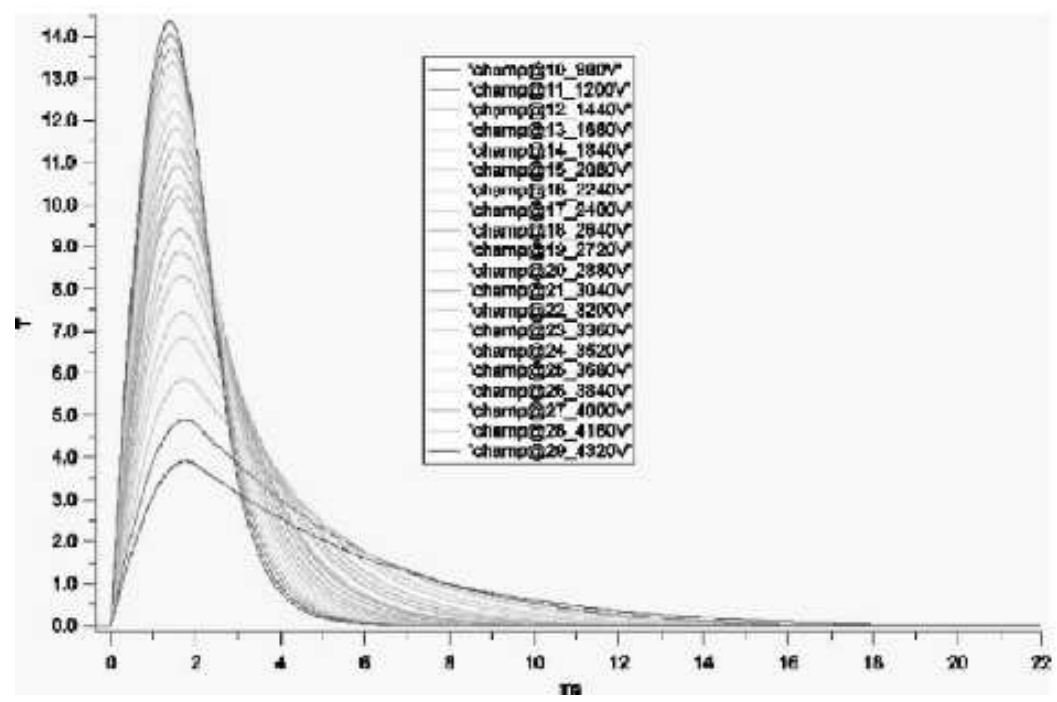

Fig. 15. Magnetic field delivered by the X-coil depending on time and for different supply voltages

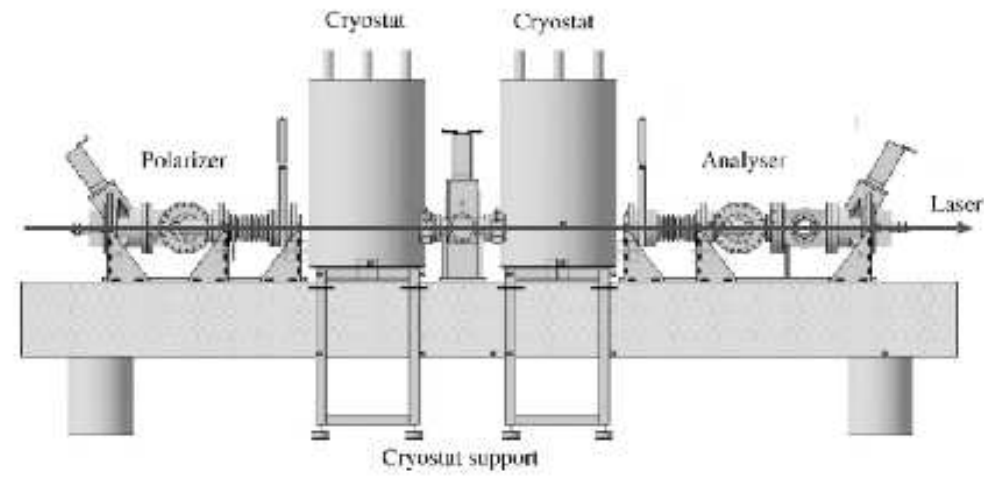

Fig. 16. Schematic view of the experimental set up of the BMV project

2006 was a crucial year for the project. In May that year the experiment will start to be set up at LNCMP. First with just one magnet in place, then, when test runs will be completed, with two magnets in place. This configuration corresponds to $40 \mathrm{~T}^{2} \mathrm{~m}$. A new set of mirrors is in preparation at LMA. The clean environments, already used at LCAR, moved in the experimental 


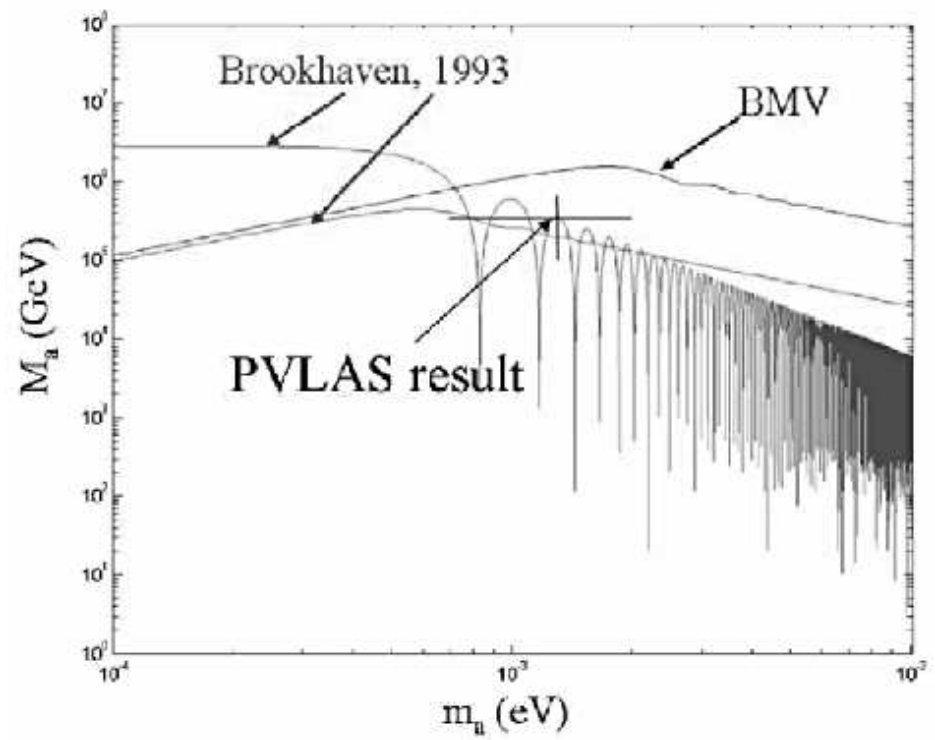

Fig. 17. Limits on the inverse coupling constant $M_{a}$ in the $m_{a}$ vs. $M_{a}$ plane for an axion-like particle given for one day of operation (20 magnet pulses) assuming a zero ellipticity measurements

clean room at LNCMP will allow to exploit at their best these new mirrors. Finesse greater than 200000 is expected. The sensitivity should also be at least $10^{-8} \times 1 / \sqrt{(H z)}$, thanks to the high central frequency of the modulated effect.

Fig. 3.2 represents the exclusion region in the $m_{a}$ vs. the inverse coupling constant $M_{a}$ plane, given for one day of operation (20 magnet pulses) and assuming a zero ellipticity measurement, based on the experimental parameters given above. The red curves indicate the Brookhaven (BFRT) result (35), the blue line the BMV projected result, and finally the black cross the region of values that could explain the PVLAS results. This interpretation would thus be excluded by a zero ellipticity measurement with the BMV setup.

\section{The OSQAR experiment at CERN (2-in-1 Experiment)}

Because of the strong transverse magnetic field, required to obtain measurable effects, an ideal implementation to investigate simultaneously the magnetooptical properties of the quantum vacuum and the photon regeneration effect, are within long superconducting accelerator dipolar magnets, such as the ones developed and manufactured for the Large Hadron Collider (LHC). The reuse of recently decommissioned $15 \mathrm{~m}$ long twin aperture LHC superconducting magnet prototypes, providing a transverse magnetic field $B=9.5 \mathrm{~T}$, offers a 


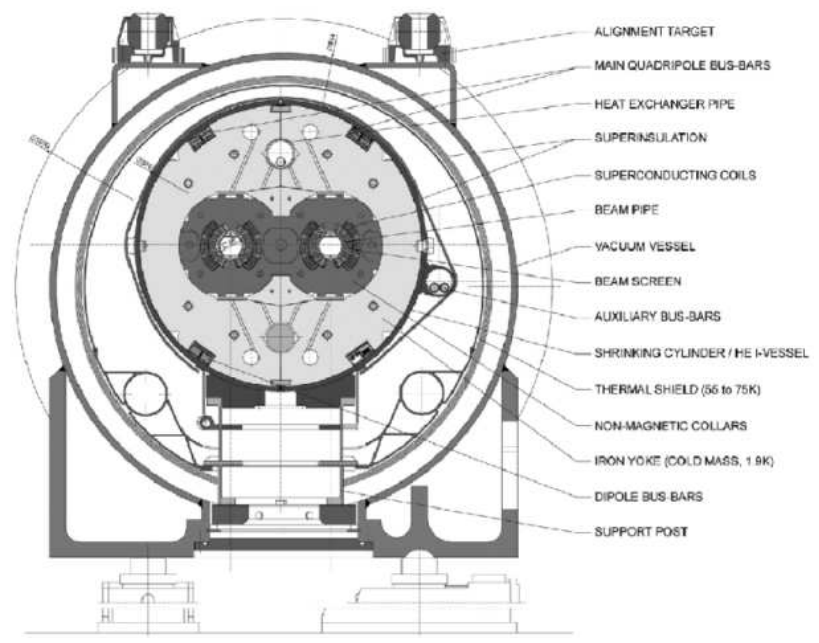

Fig. 18. Cross section of a LHC superconducting main dipole magnet housed inside its cryostat

unique opportunity for the construction of a new powerful two-in-one experiment, based on optical techniques (48). The cross section of such dipole magnet is shown in Fig. 18.

Linearly polarized laser light beams will be used as probes inside vacuum chambers housed inside superconducting magnet apertures. One of the apertures will be dedicated to the measurements of the Vacuum Magnetic Birefringence (VMB) and optical absorption anisotropy whereas the other one will be used to detect the photon regeneration from axions or axion-like particles using an invisible light shining through a wall. The availability of several decommissioned LHC superconducting magnet prototypes at CERN, offers the opportunity of possible upgrades for the proposed experiments, each of them improving the sensitivity by increasing the vacuum light pass in the transverse magnetic field with the connection of additional magnets.

Measurement of the Vacuum Magnetic Birefringence (VMB)

A first version of the experimental configuration, to measure very small optical birefringence, was proposed by (48) and is shown in Fig. 19. It is based on a Fabry-Pérot cavity of finesse equal to $10^{3}-10^{4}$ and a novel measurement method, using a double path of light through a half-wave plate, mounted in a high-speed rotation stage. The initial linear polarization state of the laser beam can be modulated typically in the $\mathrm{kHz}$ range. After the second pass through the half-wave plate, the laser beam will retrieve to the first order, its linear polarization state with the small VMB rotation angle, induced by the vacuum and submitted to the transverse $B$ field inside the optical cavity. Then a quarter-wave plate will convert the quasi-linear polarized laser 


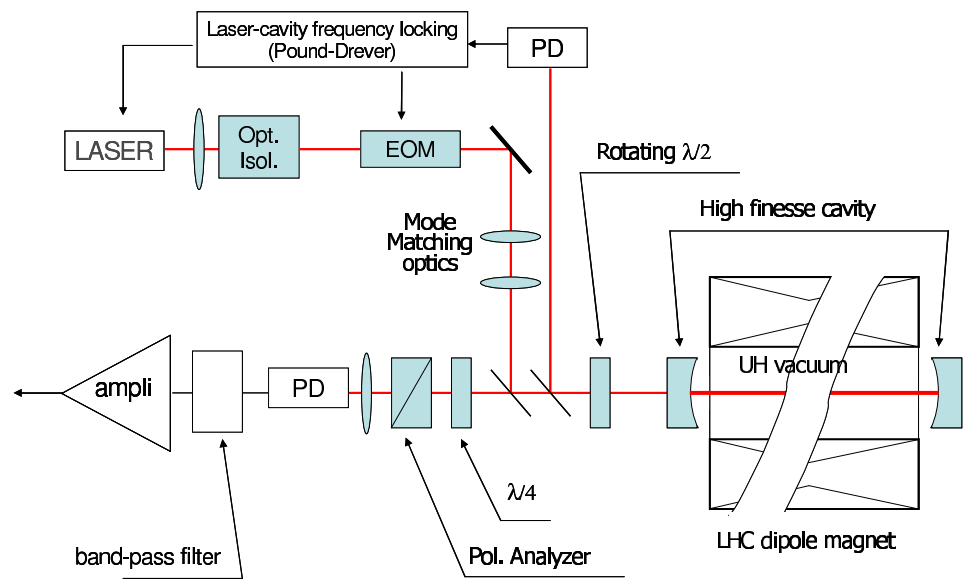

Fig. 19. Optical scheme for the measurements of the vacuum magnetic birefringence adapted from (48) with inputs from D. Romanini and L. Duvillaret

beam coming out from the cavity into a quasi-circular one. Finally, a polarizer will ensure a linear and optimal conversion of the induced ellipticity into a power modulation of the laser beam. By replacing the half-wave rotating plate with an electro-optic modulator, it can be expected to work in the $\mathrm{MHz}$ range for the modulation and the detection. This constitutes one of the alternative solutions studied at present to improve the VMB measurements. For the phase- 1 of this project, the sensitivity is expected to reach the state of the art, i.e., $10^{-9}-10^{-8} \mathrm{rad} / \sqrt{\mathrm{Hz}}$. An improvement of at least two orders of magnitude of the present reference results given by the BFRT collaboration (51), concerning the VMB and absorption anisotropy, is expected together with the measurement for the first time of the QED prediction (48).

\section{The Photon Regeneration Experiment}

To complement and cross-check the VMB measurements, the photon regeneration experiment will be integrated in the second aperture of the $15 \mathrm{~m}$ long LHC superconducting magnet. The principle of this experiment is schematized in Fig. 20. A high finesse Fabry-Pérot cavity, inserted inside a part of the dipole magnetic field region, is used as an axion source and a photomultiplier with a proper magnetic shield, or an avalanche photodiode, as an optical detection system. The optical barrier will intercept all photons not converted into axions and the detection of any photon at the same wavelength as the laser beam can be interpreted as an axion to photon reconversion inside the regeneration region. A chopper can be used for a synchronous photon counting with the chopped laser beam, to improve the background rejection. When the magnetic field is switched off, the same measurements can be repeated to detect the possible mixing effect between photons and paraphotons. 


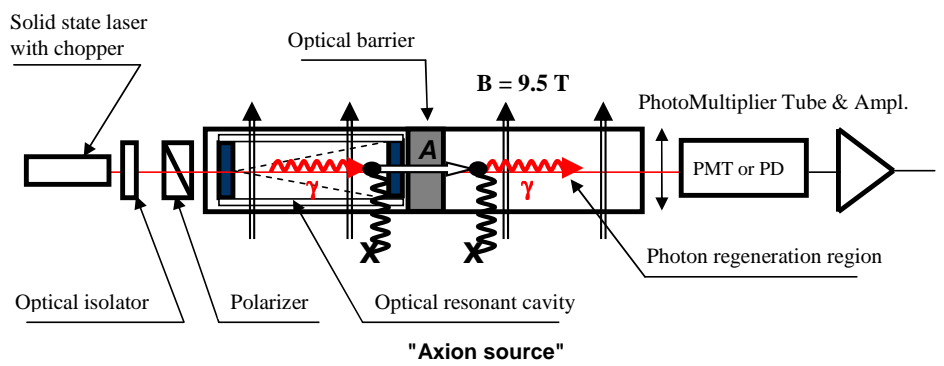

Fig. 20. Scheme of the photon regeneration experiments

With a Nd:YAG laser, an optical beam power as large as 100-1000 W can be obtained at the wavelength $\lambda=1064 \mathrm{~nm}$. The optimum for the photon regeneration experiment is obtained for an optical cavity and a regeneration region both immersed in the same magnetic field integral. Assuming as a first step the use of a single LHC dipole with a $7 \mathrm{~m}$ long regeneration region and an optical cavity with a finesse of $10^{4}-10^{5}$ of the same length, the photon counting rate given by the BFRT collaboration (51) can be improved by a factor of about $10^{8}$. This corresponds to a limit for the coupling constant to two photons $1 / M$ of about $10^{-9} \mathrm{GeV}^{-1}$, i.e., an improvement by more than 2 orders of magnitude with respect to the BFRT results. The lost of coherence in the axion to photon conversion will prevent to probe axions with a mass typically larger than $0.4 \mathrm{meV}$ at the lower value of $1 / M$. During the preparatory phase of this photon regeneration experiment, the focus will be given to the check of PVLAS results (52). Preliminary estimates show, that this objective can be achieved at $95 \%$ confidence level with an integration time of about 1 hour, assuming 1000 reflections in a cavity of $1 \mathrm{~m}$ long in a $9 \mathrm{~T}$ field and an optical power of about $1 \mathrm{~W}$.

\section{Search for Kaluza-Klein Axions with TPCs}

Higher dimensional axionic theories that include $\delta$ extra spatial dimensions to the $(3+1)$ dimensions of the Minkowski space-time predict, that the axion field can propagate in these additional dimensions (see Chap. ?? for an introduction). An important consequence of such so called Kaluza-Klein (KK) axion models would be, that the axion field would decouple from the PecceiQuinn energy scale and aquire an infinite tower of mass eigenstates. The mass spacing between individual eigenstates would then be $\propto 1 / R$, where $R$ is the compactification radius of the extra dimensions. For the corresponding axion mass eigenvalues then follows

$$
m_{a 0} \approx m_{\mathrm{PQ}} \text { and } m_{a n} \approx \frac{n}{R} \text { with } n=1,2,3, \ldots
$$



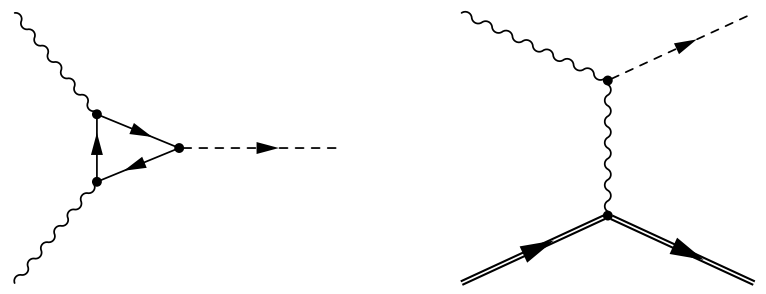

Fig. 21. Feynman diagram for axion production by photon coalescence (left) and the Primakoff effect (right)

Taking actual limits on the Peccei-Quinn scale into account, the lifetime of the Peccei-Quinn axion with respect to a $a \rightarrow \gamma \gamma$ decay calculates from

$$
\tau_{a}=\frac{64 \pi}{g_{a \gamma \gamma}^{2} m_{a}^{3}}
$$

to $10^{27}$ years $\lesssim \tau_{a} \lesssim 10^{42}$ years, which is several orders of magnitude longer compared to the age of our universe $\left(13.7 \times 10^{9}\right.$ years $\left.(61)\right)$. Particles which decay with such long lifetimes are not observable with actual experimental techniques. Taking (35) and (36) into account and setting $m_{a}=m_{a 0}$, it is obvious that the lifetime of the more massive KK-axion states is significantly shorter, e.g., we get a lifetime of $2.7 \times 10^{7}$ years $\lesssim \tau_{a} \lesssim 2.7 \times 10^{14}$ years for the $10 \mathrm{keV}$ mode. In this case, the experimental observation of the axion di-photon decay becomes feasible, as will be shown in the following sections.

\subsection{Gravitationally Trapped Solar KK-Axions}

A potential source of KK-axion would be the hot plasma of the Sun, were axions can be produced by either the Primakoff conversion or photon coalescence (see Fig. 21). The total solar axion luminosity from both contributions is given by (64)

$$
L_{a}=A L_{\odot}\left(\frac{g_{a \gamma \gamma}}{10^{-10} \mathrm{GeV}^{-1}}\right)^{2}\left(\frac{R}{\mathrm{keV}^{-1}}\right)^{\delta},
$$

with a normalization factor $A(A=0.067 / 0.12$ for axions produced by photon coalescence/Primakoff effect), the solar luminosity $L_{\odot}$, and the number of extra dimensions $\delta$. Most of these axions would freely stream out of the Sun and leave the solar system before they decay. Nevertheless, a small fraction of axions would have velocities smaller than the escape velocity $v_{\text {esc }}$ and could be trapped in the gravitational potential of the Sun. These trapped KK-axions would revolve the Sun on Keplerian orbits as shown in Fig. 22 until they decay. Since axions produced by the Primakoff effect are relativistic $\left(v \gg v_{\text {esc }}\right)$, their contribution to the overall number density of trapped axions is by a factor of $10^{3}$ smaller compared to axions produced by photon coalescence. Depending 

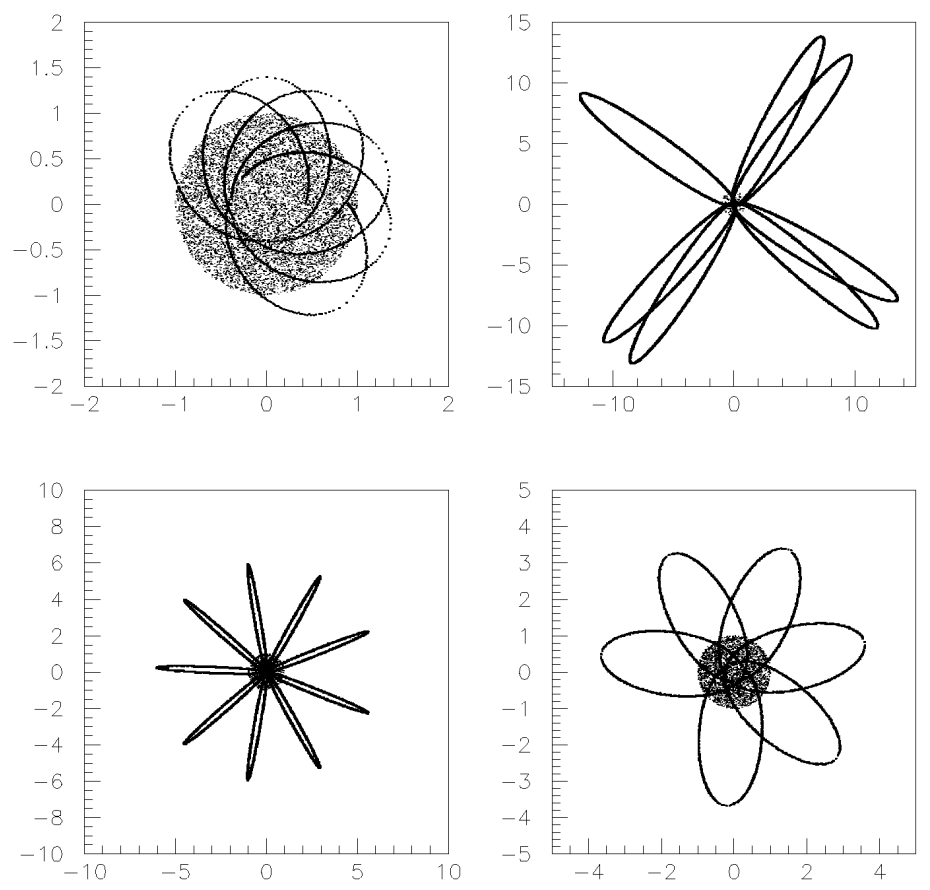

Fig. 22. Keplerian orbits of Kaluza-Klein axions trapped in the gravitational potential of the Sun. The $\mathrm{x}$ - and $\mathrm{y}$-axis indicate the distance from the Sun in units of solar radii (adapted from (64) with permission)

on the KK-axion lifetime $\tau_{a}$ the number density of trapped axions would evolve with time according to (64)

$$
N_{a}(t)=R_{a} \tau_{a}\left(1-\exp ^{-t / \tau_{a}}\right)
$$

where $R_{a}$ is the axion production rate. From (38) the present KK-axion number density can be calculated depending on the distance to the Sun, as well as the axion number density we would expect on Earth today. The resulting mass spectrum of trapped KK-axions is shown in the left part of Fig. 23 for both production processes. Assuming that KK-axions decay into two coincident photons with identical energy $E_{\gamma}=m_{a} / 2$, the decay spectrum is given by (63)

$$
\frac{\mathrm{d} R}{\mathrm{~d} m_{a}}=\frac{g_{a \gamma \gamma}^{2}}{64 \pi} n_{0} m_{a}^{3} f\left(m_{a}\right)
$$

where $f\left(m_{a}\right)$ is the trapped axion mass spectrum and $n_{0}$ the present total KK-axion number density. The right part of Fig. 23 shows the axion decay spectrum resulting from (39) on Earth depending on energy. Integrating over all energies, yields a total KK-axion decay rate of 

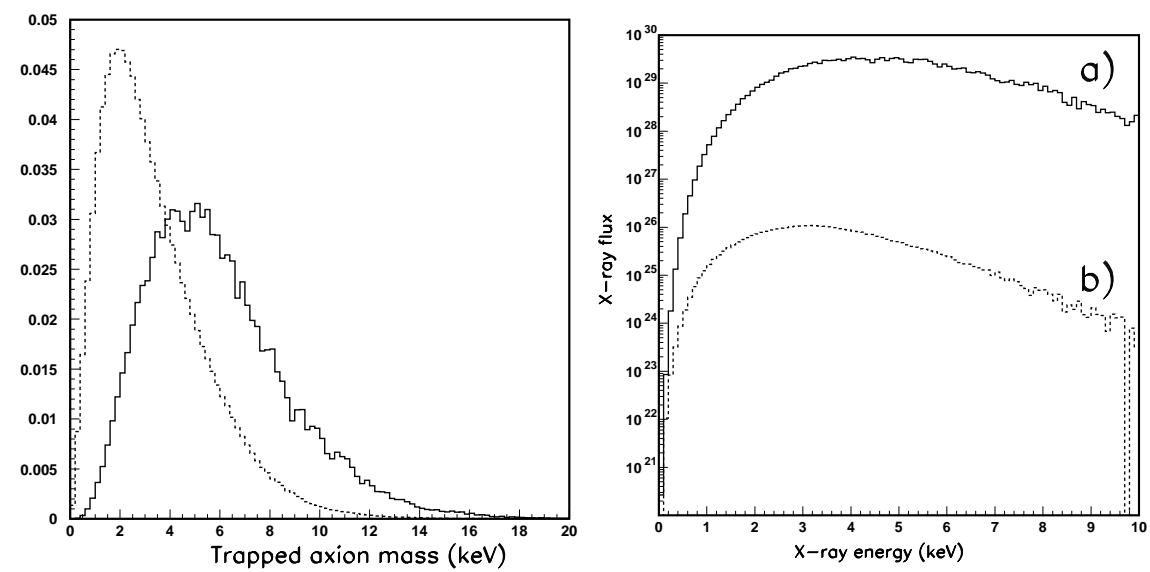

Fig. 23. Left: Mass spectrum of KK-axions trapped in the gravitational potential of the Sun which cross the orbit of the Earth. The solid line represents the mass distribution for axions produced via photon coalescence and the dashed line the mass distribution for axions produced via the Primakoff effect. Please note that the area of both distributions is normalized to unity. Right: The corresponding X-ray spectrum resulting from axion to two photon decay of axions produced via a) photon coalescence and b) the Primakoff effect (adapted from (64))

$$
R=2.5 \times 10^{11}\left(\frac{g_{a \gamma \gamma}}{\mathrm{GeV}^{-1}}\right)^{2}\left(\frac{n_{0}}{\mathrm{~m}^{-3}}\right) \mathrm{m}^{-3} \mathrm{day}^{-1}
$$

\subsection{KK-Axion Detection with Large TPCs}

The possibility that such a local axion population exists, motivates an experimental search for KK-axions. To estimate the minimum sensitivity necessary to be able to observe KK-axion decays, the expected axion di-photon decay rate can be calculated from (40). Assuming a local axion number density of $n_{0}=10^{14} \mathrm{~m}^{-3}$ and $g_{a \gamma \gamma}=9.2 \times 10^{-14} \mathrm{GeV}^{-1}$ (see $(62 ; 64)$ for details), an axion decay rate of $R=0.21 \mathrm{~m}^{-3}$ day $^{-1}$ is expected. It is obvious from this estimate that a detector with a large sensitive volume and low background is required in order to be sensitive to the resulting low count rates. In addition the detector must be able to separate the two coincident axion decay photons with an energy of $E_{\gamma}=m_{a} / 2 \approx$ few $\mathrm{keV}$ which are emitted back to back. Solid and liquid state detectors like NaI, Ge, or Xe are disfavored for this purpose. The mean free path of X-rays in, e.g., Ge is of the order of a few $\mu \mathrm{m}$, consequently the decay photons would be indistinguishable from the detector background. Instead, a large volume Time Projection Chamber (TPC) operated underground and at low pressure similar to the DRIFT II detectors (65) would be an ideal system for such an experiment. In (63) the 


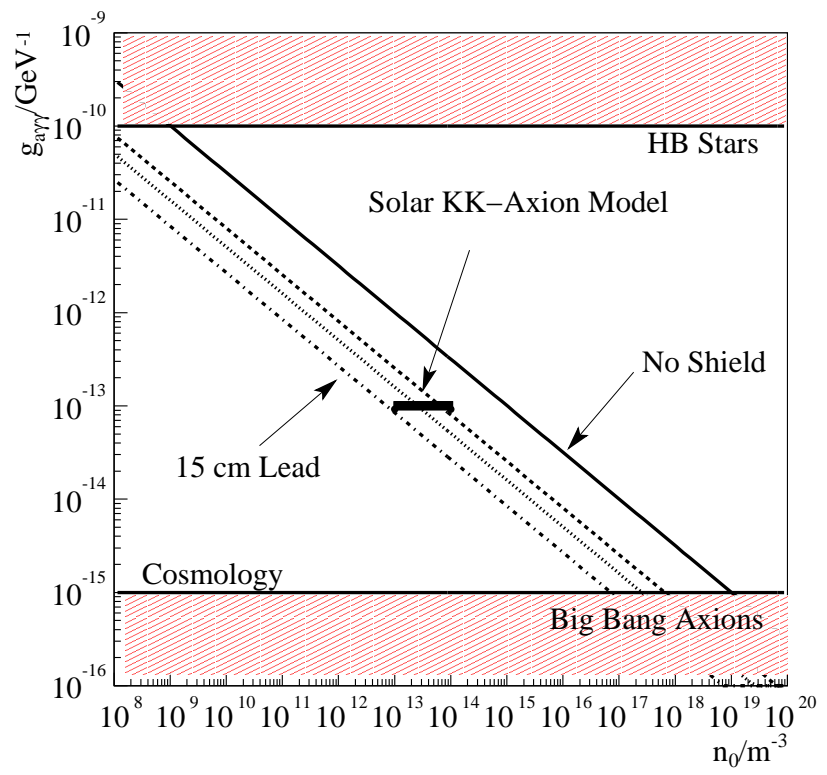

Fig. 24. Sensitivity estimate for a $1 \mathrm{~m}^{3} \mathrm{TPC}$ detector operated with $\mathrm{CS}_{2}$ as gas for different detector shield configuration. In addition exclusion contours from astrophysical considerations are shown. The parameter range predicted from the solar KK-axion model of (64) is marked by the black box (adopted from (63))

sensitivity and background rejection efficiency has been estimated based on Monte-Carlo simulations for a $1 \mathrm{~m}^{3}$ DRIFT II type detector operated with $\mathrm{CS}_{2}$ as detection gas. The resulting sensitivity is shown in Fig. 24 for realistic KK-axion model parameters and for different detector configurations. According to these results, a DRIFT II type TPC detector shielded with $15 \mathrm{~cm} \mathrm{~Pb}$ would be sufficient, to fully probe the predicted parameter range of the model presented by (64) (the range of the model parameters is indicated as a black box in Fig. 24). At present it seems feasible to realize a detector system at moderate cost by upgrading a DRIFT II detector similar to the one shown in Fig. 25. This would require additional shielding and a better spatial resolution of the detector in order to reduce the detector background and to be able to spatially resolve the secondary electrons produced by the two KK-axion decay photons.

\section{Collider Bounds on Scalars and Pseudoscalars}

If pseudoscalar particles couple to photons and gluons, it is possible to constraint their couplings and mass by looking at processes where a pseudoscalar particle is involved. The processes we consider here are $e^{+} e^{-} \rightarrow \gamma+\mathbb{E}_{T}$ for 


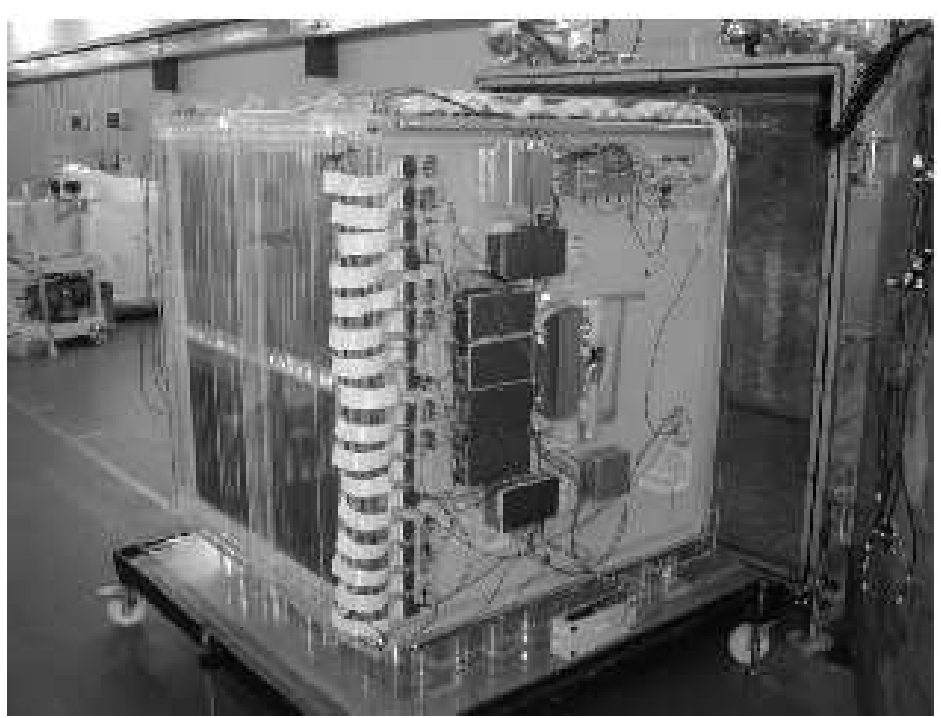

Fig. 25. Image of the DRIFT II detector during installation in the Boulby underground laboratory

$e^{+} e^{-}$colliders, and $p p$ or $p \bar{p} \rightarrow$ single jet $+\mathbb{E}_{T}$ for hadron colliders. One characteristic of the dimension five amplitudes considered here is that the cross section is independent of the center of mass energy at high energies. This follows from a dimensional analysis: the interaction $g \phi F \wedge F$, where $\phi$ is the pseudoscalar field, has a coupling constant $g$ with a dimension of inverse mass. Therefore $2 \rightarrow 2$ processes, involving the production of a single $\phi$ particle, will have a cross section

$$
\frac{\mathrm{d} \sigma}{\mathrm{d} \Omega}=g^{2} f(s / t),
$$

where $f(s / t)$ is a function which depends only on the angle. Specifically, the couplings we are interested are the following

$$
L \propto \frac{\alpha_{s}}{16 \pi f} \phi G_{\mu \nu}^{b} \tilde{G}^{b \mu \nu},
$$

for gluons, and

$$
L \propto \frac{g_{a \gamma \gamma}}{8} \phi F_{\mu \nu} \tilde{F}^{\mu \nu}
$$

for photons. Hadron colliders are more effective in providing limits for axiongluon couplings and $e^{+} e^{-}$colliders for axion-photon couplings.

\subsection{Bounds from Hadron Colliders}

Here the results for a two experiments in hadron colliders are summarized, for a more detailed analysis we refer to (53). Using the data from the DØ experiment at Fermilab a bound on $f$ can be set to 


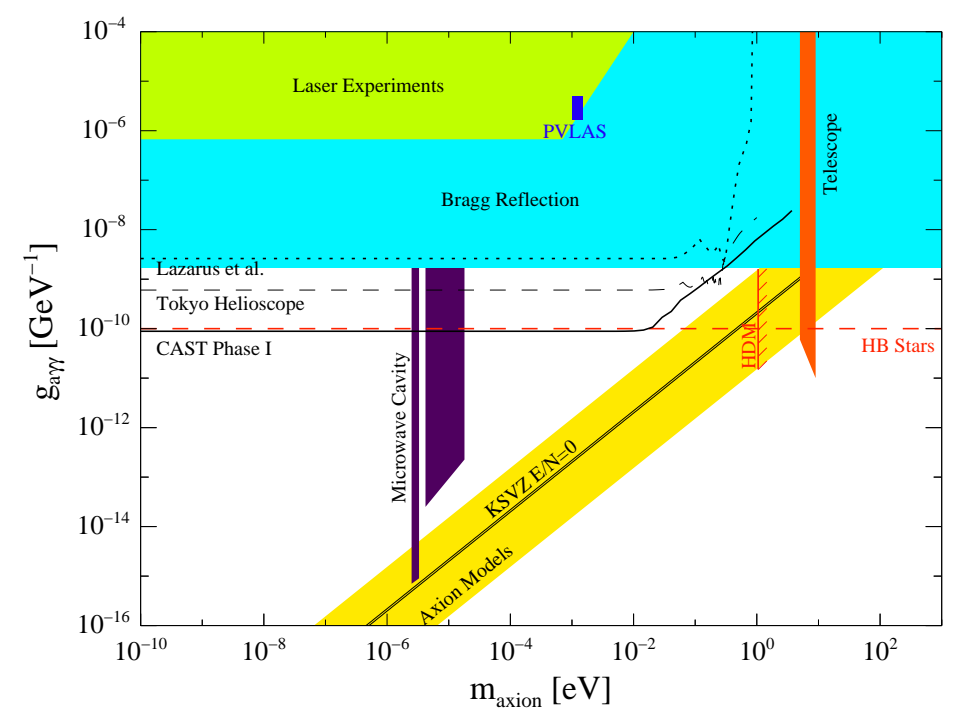

Fig. 26. Exclusion plots in the $g_{a \gamma \gamma}$ versus $m_{a}$ parameter space. Limits derived with different experimental techniques are shown. Limits from DAMA, SOLAX, and COSME are denoted as "Bragg Reflection" (24; 25; 26). In addition, the best limits from laser experiments, microwave cavity experiments, telescope searches, helioscope searches marked as "Lazarus et al.", "Tokyo Helioscope", and CAST $(5 ; 8 ; 11 ; 13 ; 12)$, and the best astrophysical limits are shown $(19 ; 20)$. The region predicted by theoretical models is marked as "Axion Models" $(E / N-1.95)$. The vertical line "HDM" indicates the hot dark matter limit for hadronic axions (66)

$$
f>35 \mathrm{GeV} .
$$

As for future and ongoing experiments, the Large Hadron Collider (LHC) at CERN is expected to improve the bound on $f$ to

$$
f_{\mathrm{LHC}}>1300 \mathrm{GeV} .
$$

\subsection{Bounds from $e^{+} e^{-}$Colliders}

More interesting are the limits that can be obtained for the photon coupling to axions in $e^{+} e^{-}$colliders. Several experiments during the past and future have been analyzed to determine the bound which could be obtained assuming the data are consistent with the standard model backgrounds. A bound $g_{a \gamma \gamma}<5.5 \times 10^{-4} \mathrm{GeV}^{-1}$ was obtained using $e^{+} e^{-}$collider data from ASP (54) (55). Since the amplitudes are independent of energy, the bounds can be improved mainly by increasing the total luminosity. An analysis of the combined data from LEP at ALEPH, OPAL, L3, and DELPHI would yield the more restrictive bound: 


$$
g_{a \gamma \gamma}<1.5 \times 10^{-4} \mathrm{GeV}^{-1},
$$

for $m_{\phi}<65 \mathrm{MeV}$. For the PEP-II $e^{+} e^{-}$collider, current integrated luminosity gives

$$
g_{a \gamma \gamma}(\mathrm{PEPII})<8.9 \times 10^{-6} \mathrm{GeV}^{-1},
$$

for $m_{\phi}<0.12 \mathrm{GeV}$. A similar bound can be obtained from KEKB $e^{+} e^{-}$ collider. With the current integrated luminosity the bound would be

$$
g_{a \gamma \gamma}(\mathrm{KEKB})<8.2 \times 10^{-6} \mathrm{GeV}^{-1},
$$

for $m_{\phi}<0.13 \mathrm{GeV}$. The expected total luminosity for KEKB is at least twice the current total, which would improve the bound to $g_{a \gamma \gamma}<5.9 \times 10^{-6} \mathrm{GeV}^{-1}$. Finally, for the Super KEKB upgrade to KEKB is expected to produce $10^{7} \mathrm{pb}^{-1}$ per year. After two years, this would improve the bound to

$$
g_{a \gamma \gamma}(\mathrm{KEKB})<1.9 \times 10^{-6} \mathrm{GeV}^{-1},
$$

which would rule out most of the parameter space favored by PVLAS.

\section{Summary and Outlook}

In this paper we reviewed different experimental searches for axions and axionlike particles which are actually running or planned to take date in the near future. Already existing upper limits on the axion to photon coupling strength

$g_{a \gamma \gamma}$ in the mass range of $1 \times 10^{-10} \mathrm{eV} \lesssim m_{a} \lesssim 1 \times 10^{2} \mathrm{eV}$ derived with different experimental techniques are summarized in Fig. 26. In addition the parameter range that can be derived from the PVLAS result is indicated by a rectangular box marked as "PVLAS".

It was not our intention to give a complete review of all existing experimental techniques to search for axions, this would be beyond the scope of an article like ours. Instead, we focused on the topics which were addressed during the Joint ILIAS-CAST-CERN Axion Training at CERN. The common factor of all experiments is the inspired techniques used, which are extremely challenging and innovative.

The fact that the PVLAS collaboration has reported a positive signal that could be interpreted as a signature of an axion-like particle has boosted a race, to build a new set of laser-based experiments. We are convinced that the following years are going to be very exciting.

\section{Acknowledgment}

We acknowledge support from the Virtuelles Institut für Dunkle Materie und Neutrinophysik - VIDMAN (Germany). Furthermore, the authors acknowledge the helpful discussions within the network on direct dark matter detection of the ILIAS integrating activity of the European Union (contract 
number: RII3-CT-2003-506222). Parts of this work has been performed in the CAST collaboration, we thank our colleagues for their support. The CAST project was supported by the Bundesministerium für Bildung und Forschung (BMBF) under the grant number 05 CC2EEA/9 and 05 CC1RD1/0.

\section{References}

[1] S. De Panfilis et al., "Limits on the abundance and coupling of cosmic axions at $4.5 \mu \mathrm{eV}<M(A)<5.0 \mu \mathrm{eV}$ ", Phys. Rev. Lett. 59, 839 (1987); W. U. Wuensch et al., "Results of a laboratory search for cosmic axions and other weakly coupled light particles", Phys. Rev. D 40, 3153 (1989).

[2] C. Hagmann, P. Sikivie, N. S. Sullivan and D. B. Tanner, "Results From A Search For Cosmic Axions", Phys. Rev. D 42, 1297 (1990).

[3] S. J. Asztalos et al., "An improved RF cavity search for halo axions", Phys. Rev. D 69, 011101 (2004) [astro-ph/0310042].

[4] M. Tada et al., "CARRACK II: A new large-scale experiment to search for axions with Rydberg-atom cavity detector", Nucl. Phys. Proc. Suppl. 72,164 (1999).

[5] S. Andriamonje [CAST Collaboration], "An improved limit on the axion - photon coupling from the CAST experiment", J. Cosmol. Astropart. Phys., 010 (2007), [hep-ex/0702006].

[6] J. N. Bahcall and M. H. Pinsonneault, "What do we (not) know theoretically about solar neutrino fluxes", Phys. Rev. Lett. 92, 121301(1) (2004), [astro-ph/0402114].

[7] P. Sikivie, "Experimental tests of the invisible axion", Phys. Rev. Lett. 51,1415 (1983);

(E) ibid. 52, 695 (1984).

[8] D. M. Lazarus, G. C. Smith, R. Cameron, A. C. Melissinos, G. Ruoso, Y. K. Semertzidis and F. A. Nezrick, "A Search for solar axions", Phys. Rev. Lett. 69, 2333 (1992).

[9] A. A. Anselm, "Arion $\leftrightarrow$ Photon Oscillations in a Steady Magnetic Field. (In Russian)," Yad. Fiz. 42, 1480 (1985).

[10] K. van Bibber, P. M. McIntyre, D. E. Morris and G. G. Raffelt, "A Practical Laboratory Detector For Solar Axions", Phys. Rev. D 39, 2089 (1989).

[11] S. Moriyama, M. Minowa, T. Namba, Y. Inoue, Y. Takasu and A. Yamamoto, "Direct search for solar axions by using strong magnetic field and X-ray detectors", Phys. Lett. B 434, 147 (1998) [hep-ex/9805026].

[12] Y. Inoue, T. Namba, S. Moriyama, M. Minowa, Y. Takasu, T. Horiuchi and A. Yamamoto, "Search for sub-electronvolt solar axions using coherent conversion of axions into photons in magnetic field and gas helium", Phys. Lett. B 536, 18 (2002) [astro-ph/0204388]. 
[13] S. Moriyama, M. Minowa, T. Namba, Y. Inoue, Y. Takasu and A. Yamamoto, "Direct search for solar axions by using strong magnetic field and x-ray detectors", Phys. Lett. B 434, 147 (1998) [hep-ex/9805026].

[14] K. Zioutas et al. [CAST Collaboration], "First results from the CERN axion solar telescope (CAST)", Phys. Rev. Lett. 94 (2005) 121301 [hepex/0411033].

[15] P. Abbon, "The Micromegas detector of the CAST experiment", New. J. Phys. submitted [physics/0702190].

[16] M. Kuster et al., "The X-ray Telescope of CAST", New. J. Phys. submitted [physics/0702188].

[17] D. Autiero, "The CAST Time Projection Chamber", New. J. Phys. submitted [physics/0702189].

[18] K. Zioutas et al. (CAST Collaboration), "First results from the CERN Axion Solar Telescope", Phys. Rev. Lett. 94, 121301 (2005) [hepex/0411033].

[19] G. G. Raffelt, "Astrophysical axion bounds", [hep-ph/0611350].

[20] Raffelt G G 1996 Stars as Laboratories for Fundamental Physics (Chicago: The University of Chicago Press).

[21] E. A. Paschos and K. Zioutas, "A Proposal for solar axion detection via Bragg scattering", Phys. Lett. B 323, 367 (1994).

[22] S. Cebrían et al., "Prospects of solar axion searches with crystal detectors", Astropart. Phys. 10, 397 (1999).

[23] R. Bernabei et al., "Performance of the $100 \mathrm{~kg} \mathrm{NaI}(\mathrm{Tl})$ set-up of the DAMA experiment at Gran Sasso", Nuovo Cim. 112, 545 (1999).

[24] A. Morales et al. [COSME Collaboration], "Particle dark matter and solar axion searches with a small germanium detector at the Canfranc underground laboratory", Astropart. Phys. 16, 325 (2002) [hep$\mathrm{ex} / 0101037]$.

[25] F. T. . Avignone et al. [SOLAX Collaboration], "Experimental search for solar axions via coherent Primakoff conversion in a germanium spectrometer", Phys. Rev. Lett. 81, 5068 (1998) [astro-ph/9708008].

[26] R. Bernabei et al., "Search for solar axions by Primakoff effect in NaI crystals", Phys. Lett. B 515, 6 (2001).

[27] K. Jahoda et al, Proc. SPIE Vol. 2808, p. 59-70, EUV, X-Ray, and Gamma-Ray Instrumentation for Astronomy VII, Oswald H. Siegmund; Mark A. Gummin; Eds.

[28] H. Davoudiasl and P. Huber, "Detecting solar axions using earth's magnetic field", Phys. Rev. Lett. 97, 121302 (2006). [hep-ph/0509293].

[29] The Suzaku team, private communication.

[30] F.S. Porter and the Suzaku Team (2005), http://www .astro.isas .ac . $\mathrm{jp} /$ suzaku/doc/suzaku_td/node9.html.

[31] K. Van Bibber, N. R. Dagdeviren, S. E. Koonin, A. Kerman and H. N. Nelson, "An Experiment To Produce And Detect Light Pseudoscalars", Phys. Rev. Lett. 59, 759 (1987). 
[32] A. Ringwald, "Axion interpretation of the PVLAS data?", J. of Phys. Conf. Ser. 39, 197 (2005) [hep-ph/0511184].

[33] E. Zavattini et al. [PVLAS Collaboration], "Experimental observation of optical rotation generated in vacuum by a magnetic field", Phys. Rev. Lett. 96, 110406 (2006) [hep-ex/0507107].

[34] K. Ehret et al., "Production and detection of axion-like particles in a HERA dipole magnet: Letter-of-intent for the ALPS experiment", Letter of Intent [hep-ex/0702023].

[35] R. Cameron et al., "Search for nearly massless, weakly coupled particles by optical techniques", Phys. Rev. D 47, 3707 (1993).

[36] R. Rabadan, A. Ringwald and K. Sigurdson, "Photon regeneration from pseudoscalars at X-ray laser facilities", Phys. Rev. Lett. 96, 110407 (2006) [hep-ph/0511103].

[37] A. Ringwald, "Fundamental Physics at an X-ray Free Electron Laser", in Proceedings of the Workshop on "Electromagnetic Probes of Fundamental Physics", edited by W. Marciano and S. White (World Scientific, Singapore, 2003), pp. 63-74 [hep-ph/0112254].

[38] "Free-electron laser at TESLA Test Facility", http://www-hasylab. desy.de/facility/fel/main.htm, "SASE FEL at the TESLA facility, phase 2",DESY-TESLA-FEL-2002-01 (2002).

[39] U. Koetz, A. Ringwald and T. Tschentscher: "Production and Detection of Axion-Like Particles at the VUV-FEL: Letter of Intent" [hepex/0606058].

[40] Catalogue "Photomultiplier Tubes and Related Products," 2003 Hamamatsu Photonics K.K., Dec. 2003 revised, http://www . hamamatsu . com.

[41] J. Barnstedt et al., "The ORFEUS II Echelle spectrometer: Instrument description, performance and data reduction", Astr. \& Astrophys. Supl. Ser. 134, 561 (1999).

[42] G. Bailly, R. Battesti, S. Batut, S. Faure, P. Ganau, J.-M. Mackowski, C. Michel, M. Nardone, L. Pinard, L. Polizzi, B. Pinto Da Souza, O. Portugall, A. Remilleux, G. L. J. A. Rikken, C. Rizzo, C. Robillard, G. Trénec, J. Vigué [The BMV Collaboration].

[43] L. Maiani, R. Petronzio, and E. Zavattini, "Effects of nearly massless, spin-zero particles on light propagation in a magnetic field", Phys. Lett. 175B, 359 (1986).

[44] W. Heisenberg and H. Euler, "Folgerungen aus der Diracschen Theorie des Positrons", Z. Phys. 98, 718 (1936);

V.S. Weisskopf, "Über die Elektrodynamik des Vakuums auf Grund der Quantentheorie des Elektrons", Mat. Phys. Medd.-K. Dan. Vidensk. Selsk. 14, 6 (1936);

J. S. Schwinger, "On gauge invariance and vacuum polarization", Phys. Rev. 82, 664 (1951).

[45] S. L. Adler, "Photon splitting and photon dispersion in a strong magnetic field", Annals Phys. 67, 599 (1971). 
[46] S. L. Adler et al., "Photon splitting in a strong magnetic field", Phys. Rev. Lett. 25, 1061 (1970).

[47] E. Gabrielli et al., "Photon propagation in magnetic and electric fields with scalar/pseudoscalar couplings: new look", Phys. Rev. D74, 073002 (2006) [hep-ph/0604143].

[48] P. Pugnat et al., J. Phys. 55 A389 (2005);

P. Pugnat et al. "Letter of Intent submitted to the CERN SPSC", CERN-SPSC-2005-034, 17th October 2005;

P. Pugnat et al., Czech. J. Phys. 56 (2006).

[49] K. van Bibber et al., "Proposed experiment to produce and detect light pseudoscalars", Phys. Rev. Lett. 59, 759 (1987).

[50] P. Sikivie, "Experimental tests of the "invisible" axion," Phys. Rev. Lett. 51, 1415 (1983), (E) ibid. 52, 695 (1984); P. Sikivie, Phys. Rev. D 322988 (1985).

[51] R. Cameron et al., "Search for nearly massless, weakly coupled particles by optical techniques", Phys. Rev. D 47, 3707 (1993).

[52] E. Zavattini et al. (PVLAS Collaboration), "Experimental observation of optical rotation generated in vacuum by a magnetic field", Phys. Rev. Lett. 96, 110406 (2006) [hep-ex/0507107].

[53] M. Kleban and R. Rabadan, "Collider Bounds on Pseudoscalars Coupling to Gauge Bosons", [hep-ph/0510183].

[54] E. Masso and R. Toldra, "On a light spinless particle coupled to photons", Phys. Rev. D 52, 1755 (1995) [hep-ph/9503293].

[55] C. Hearty et al., "Search for the anomalous production of single photons in $\mathrm{e}^{+} \mathrm{e}^{-}$annihilation at $\sqrt{s}=29 \mathrm{GeV}$ ", Phys. Rev. D 39, 3207 (1989).

[56] A. Ringwald, "Production and Detection of Very Light Bosons in the HERA Tunnel", Phys. Lett. B 569, 51 (2003).

[57] M. Ahlers: unpublished.

[58] Landolt-Börnstein, New Series, Volume 2b, Geophysics of the Solid Earth, the Moon and the Planets, pp 31-99, Springer, Berlin, 1985.

[59] E. Massó and J. Redondo, "Evading astrophysical constraints on axionlike particles", JCAP 0509, 015 (2005) [hep-ph/0504202].

[60] P. Pugnat et al., "Feasibility study of an experiment to measure the vacuum magnetic birefringence", Czech. J. Phys. 55, A389 (2005).

[61] D. N. Spergel et al. (WMAP Collaboration), "First year Wilkinson Microwave Anisotropy Probe (WMAP) observations: determination of cosmological parameters", Astrophys. J. Suppl. 148, 175 (2003) [astro$\mathrm{ph} / 0302209]$.

[62] B. Morgan, "Dark Matter Detection with Gas Time Projection Chambers", The University of Sheffield - Department of Physics and Astronomy, PhD Thesis (2004).

[63] B. Morgan et al., "Searches for Kaluza-Klein axions with gas TPCs", Astropart. Phys. 23, 287 (2005). 
[64] L. DiLella and K. Zioutas, "Observational evidence for gravitationally trapped massive axion(-like) particles", Astropart. Phys. 19, 145 (2003) [astro-ph/0207073].

[65] G. J. Alner et al., "The DRIFT-II dark matter detector: design and commissioning", Nucl. Instr. Meth. Phys. Res. A 555, 173 (2005).

[66] S. Hannestad, A. Mirizzi and G. Raffelt, "New cosmological mass limit on thermal relic axions", JCAP 0507, 002 (2005) [hep-ph/0504059]. 